\title{
Electron-Spin Structure and Metal-Ligand Bonding in Open-Shell Systems from Relativistic EPR and NMR: A Case Study of Square-Planar Iridium Catalysts
}

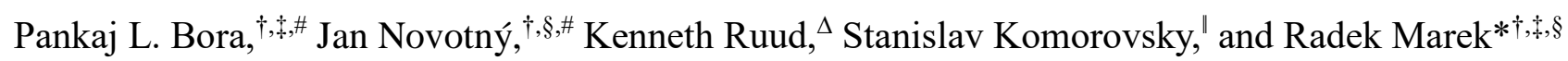

${ }^{\dagger}$ CEITEC - Central European Institute of Technology, Masaryk University, Kamenice 5/A4, CZ62500 Brno, Czechia

$\$$ Department of Chemistry and ${ }^{\S}$ National Center for Biomolecular Research, Faculty of Science, Masaryk University, Kamenice 5, CZ-62500 Brno, Czechia

$\Delta$ Hylleraas Centre for Quantum Molecular Science, Department of Chemistry, UiT - The Arctic University of Norway, N-9037 Tromsø, Norway

\| Institute of Inorganic Chemistry, Slovak Academy of Sciences, Dúbravská cesta 9, SK-84536 Bratislava, Slovakia 


\section{Abstract}

Electron and nuclear magnetic resonance spectroscopies are indispensable and powerful methods for investigating the molecular and electronic structures of open-shell systems. We demonstrate that the NMR and EPR parameters are extremely sensitive quantitative probes for the electronic spin density around heavy-metal atoms and the metal-ligand bonding. Using relativistic density-functional theory, we have analyzed the relation between the spin density and the EPR and NMR parameters in paramagnetic iridium(II/IV) complexes with a PNP pincer ligand. As the magnetic-response parameters for compounds containing $5 \mathrm{~d}$ transition metal(s) are heavily affected by spin-orbit coupling, relativistic effects must be included in the calculations. We have used a recent implementation of the fully-relativistic Dirac-Kohn-Sham (DKS) method employing the hybrid PBE0 functional and an implicit solvent model to calculate EPR parameters and hyperfine NMR shifts. The modulation of the metal-ligand bond by the trans substituent $(-\mathrm{Cl}$ or $\equiv \mathrm{N})$ and the electronic spin structure around the central metal atom and ligands are shown to be reflected in the "long-range" through-bond Fermi-contact (FC) contributions to the ligand ${ }^{13} \mathrm{C}$ and ${ }^{1} \mathrm{H}$ hyperfine couplings. Interestingly, the hyperfine coupling constant of the ligand atom $\mathrm{L}\left(A_{\mathrm{L}}\right)$ bonded directly to the iridium center changes its sign because of the dominating role of the paramagnetic spin-orbit (PSO) term. Furthermore, the electronic $g$-shift and the PSO contribution to the ligand $A_{L}$ are shown to invert their signs when nitrogen is substituted for chlorine, reflecting the different formal metal oxidation states and the change in metal-ligand bond character. A full understanding of the substituent effects is provided by using chemical bond concepts in combination with a molecular-orbital (MO) theory analysis of the second-order perturbation theory expression for the EPR parameters. Our findings are easily transferable to other systems containing $d$-block elements and beyond. Relativistic DFT calculations of magnetic-resonance parameters are expected to frequently assist in future experimental observations and the characterization of hitherto unknown unstable or exotic species.

\section{TOC/Abstract graphic}

\section{Small change?}
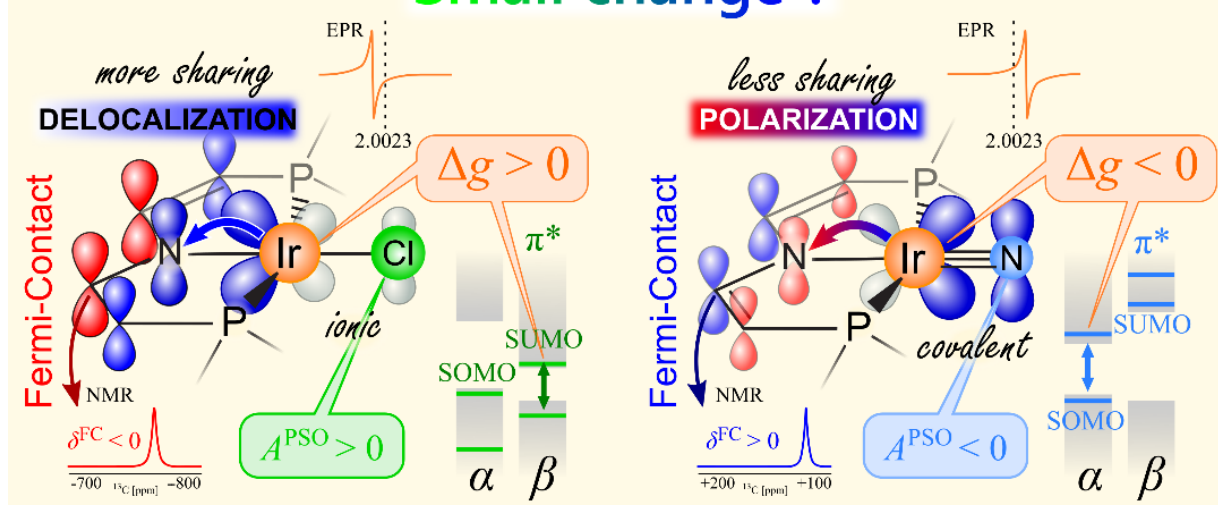


\section{INTRODUCTION}

Magnetic resonance spectroscopy is one of the most important and frequently used tools for exploring the chemical structure of any new compound. Open-shell systems can be characterized experimentally by both electron paramagnetic (EPR) and nuclear magnetic (NMR) resonance spectroscopies. Details of the electronic structure can be extracted from the EPR electronic g-tensor and hyperfine coupling tensor (A-tensor). ${ }^{1,2}$ For a system with a single paramagnetic center, $\mathbf{g}$ represents a global response property and is related to the magnetic susceptibility tensor, whereas details about the chemical bonds and electron spin structure distributed over the entire molecule can in most cases be extracted from the A-tensors of the ligand atoms. In parallel, the NMR signals of the individual atomic nuclei are significantly shifted by the nucleus-electron(s) hyperfine interaction. However, this coupling also induces a fast nuclear-spin relaxation that can result in significant broadening of the NMR signals. ${ }^{3,4}$ The hyperfine contribution to the total NMR chemical shift is temperature dependent as determined by the Boltzmann distribution over the Zeeman-split states and can be calculated using knowledge of the electronic $\mathbf{g}$ and $\mathbf{A}$ tensors, see section Theoretical Background. 5,6

Transition-metal complexes are frequently employed as catalysts in chemical transformations. ${ }^{7}$ The importance of open-shell coordination compounds of the precious metals has been highlighted in oneelectron redox reactions such as $\mathrm{C}-\mathrm{H}$ and $\mathrm{C}-\mathrm{C}$ activation or catalytic oxidation. ${ }^{8-10}$ Other transformations involve complexes with chemisorbed nitrogen atoms ${ }^{11}$ as intermediate species in the production of nitric oxide (NO) and in nitrogen-based fuel cells. ${ }^{12}$ Nitrido complexes ${ }^{13-19}$ have been specifically designed for oxidative N-N coupling reactions. Open-shell square-planar iridium amido complexes based on the PNP pincer and containing terminal $-\mathrm{Cl},-\mathrm{N}_{3}$, or $\equiv \mathrm{N}$ ligands are particularly promising candidates (Figure 1), and have been prepared and structurally characterized by diffraction experiments, EPR, and ${ }^{1} \mathrm{H}$ NMR spectroscopy. ${ }^{20,21}$ The electronic structures of these compounds were analyzed in detail in the original papers, and the oxidation state + II was assigned to iridium in compounds 1 and 2 with an $\operatorname{Ir}^{(+I I)}-\mathrm{L}^{(-\mathrm{I})}$ bond. In contrast, the formal oxidation state $+\mathrm{IV}$ was ascribed to iridium in compound 3 as shown in Figure 1, with the $\mathrm{Ir}^{(+\mathrm{IV})} \equiv \mathrm{Nl}^{(-\mathrm{III})}$ fragment having a significant amount of nitrido radical character as expressed by the resonance structure $\operatorname{Ir}^{(+\mathrm{III})}=$ N.'(-II). 
a)

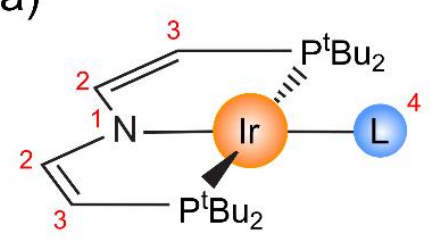

1: $\mathrm{L}=\mathrm{Cl}$

2: $L=N_{3}$

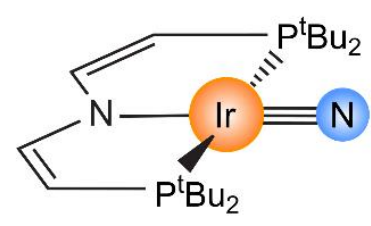

3

b)

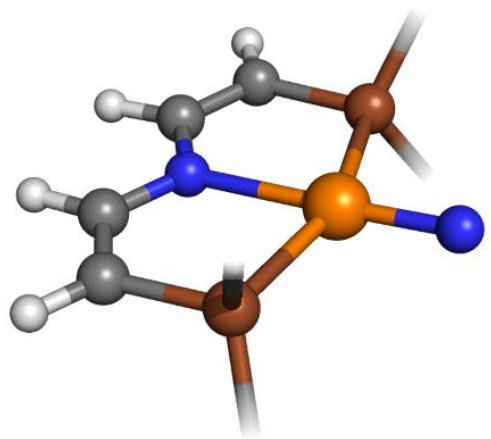

Figure 1. a) Structure and atom numbering scheme for iridium complexes 1-3 based on PNP pincer and containing terminal ligand L (highlighted in blue). b) A detail of the atomic arrangement in the square-planar core of molecule $3-\mathrm{CH}_{3}$ groups of the tert-butyl ( ${ }^{\mathrm{t}} \mathrm{Bu}$ ) fragments are omitted for clarity.

However, no quantitative analysis of the magnetic response parameters was attempted for these systems. This is partly due to the fact that such open-shell $5 \mathrm{~d}$ transition-metal complexes represent a challenge for computational chemistry because of the large effects of spin-orbit (SO) coupling. The authors of the original papers ${ }^{20,21}$ even noted that "with large SO coupling spin is not a good quantum number to quantitatively describe electronic structure ${ }^{22 \%}$.

In this contribution, we perform a detailed analysis of the EPR and NMR properties of paramagnetic square-planar Ir complexes differing in the terminal ligand L, as shown in Figure 1. Because of the size and nature of the systems investigated, our relativistic calculations are restricted to the level of Density-Functional Theory (DFT). Large and polarizable basis sets are required to achieve the accuracy necessary to allow for a quantitative comparison with experimental data. Variational inclusion of spin-orbit coupling is essential to quantitatively reproduce experimental data, but we will also show that a semi-quantitative analysis can nevertheless be undertaken using second-order perturbation theory. The goal of this study is to unravel how the different types of metal-ligand bonding and electronic spin structure around the central metal atom are reflected in the magnetic resonance parameters, and to rationalize this relation in terms of bonding concepts and MO theory. 


\section{THEORETICAL BACKGROUND}

The fundamental parameters of electron paramagnetic resonance, the electronic g-tensor and hyperfine A-tensor, are derived from the molecular topology and the distribution of electron spin in the system. The electronic $g$-shift is the isotropic characteristic that represents the departure of the molecular electron-resonance frequency from the free-electron value ( 2.0023). In complex molecular systems, the electronic shielding/deshielding is typically highly anisotropic in different molecular directions relative to the applied magnetic field and is described by $\Delta \mathbf{g}-$ the second-rank $\mathbf{g}$-shift tensor. In the case of metal-centered radicals, $\mathbf{g}$ is derived from the electronic structure around the central metal atom.

\section{Electronic g-tensor}

We recall that the $\mathbf{g}$-shift tensor $(\Delta \mathbf{g})$ is a purely relativistic property, arising primarily from the spinorbit coupling. The total $\mathbf{g}$ in four-component relativistic theory, used for the quantitative calculations in this work, can be expressed as an inner product of the orbital-Zeeman (OZ), spin-Zeeman (SZ), and relativistic (REL) operators with the four-component wave function

$$
\mathbf{g}=g_{\mathrm{e}}+\Delta \mathbf{g}=g_{\mathrm{e}}+\Delta \mathbf{g}^{\mathrm{OZ}}+\Delta \mathbf{g}^{\mathrm{SZ}}+\Delta \mathbf{g}^{\mathrm{REL}}
$$

where $g_{e}$ is the value of $g$ for the Zeeman effect of a free electron $(\sim 2.0023) . \Delta \mathbf{g}^{\mathrm{REL}}$ is a relativistic contribution without direct analog in standard theories for the electronic g-tensor. Note that every contribution to the g-shift in Eq. (1) contains both scalar-relativistic and spin-orbit effects. The orbitalZeeman contribution, $\Delta \mathbf{g}^{\mathrm{OZ}}$, frequently dominates, or at least determines the trends of, the total $\Delta \mathbf{g}$ value in a series of compounds, as shown in the Table S1 in Supporting Information also in the case of 1 and 3.

The leading-order correction to the orbital-Zeeman term $\left(\Delta \mathbf{g}^{\mathrm{OZ}}\right)$ originates from the interaction of the external magnetic field and the spin-orbit coupling, termed $\Delta \mathbf{g}^{\mathrm{SO} / \mathrm{OZ}} \cdot \cdot^{23-25}$ The second-order perturbation expression for the $\mathbf{g}^{\mathrm{SO} / \mathrm{OZ}} \operatorname{term}^{24}$ can for the purpose of a semi-quantitative MO analysis be approximated by

$$
\Delta \mathbf{g}^{\mathrm{SO} / \mathrm{OZ}} \approx \sum_{i=1}^{\mathrm{occ}} \sum_{a=1}^{\mathrm{vac}}\left(\frac{\left\langle\varphi_{i}^{\alpha}\left|r_{M}^{-3} \hat{\boldsymbol{l}}^{M}\right| \varphi_{a}^{\alpha}\right\rangle\left\langle\varphi_{a}^{\alpha}\left|\hat{\boldsymbol{l}}^{M}\right| \varphi_{i}^{\alpha}\right\rangle}{\varepsilon_{i}^{\alpha}-\varepsilon_{a}^{\alpha}}-\frac{\left\langle\varphi_{i}^{\beta}\left|r_{M}^{-3} \hat{\boldsymbol{l}}^{M}\right| \varphi_{a}^{\beta}\right\rangle\left\langle\varphi_{a}^{\beta}\left|\hat{\boldsymbol{l}}^{M}\right| \varphi_{i}^{\beta}\right\rangle}{\varepsilon_{i}^{\beta}-\varepsilon_{a}^{\beta}}\right)
$$

where $\varphi_{i}^{X}\left(\varphi_{a}^{X}\right)$ denotes occupied (vacant) molecular orbitals, $\varepsilon_{i}^{X}\left(\varepsilon_{a}^{X}\right)$ stands for occupied (vacant) one-electron energies, and $X=\alpha, \beta$ indicates the orientation of the spin variable. This Ramsey-type formula describes the coupling between individual occupied-vacant MO-pairs ${ }^{1}$ through the approximate one-electron spin-orbit operator, $r_{M}^{-3} \hat{\boldsymbol{l}}^{M}$ (for details, see section Methods), and the 
angular momentum operator, $\hat{\boldsymbol{l}}^{M}$, both centered on the metal atom. $r_{M}$ represents the position of the electron relative to the metal atom $\mathrm{M}$. The SO operator is of a highly local nature and therefore couples $\mathrm{MO} \leftrightarrow \mathrm{MO}^{*}$ pairs only where both MOs have a significant admixture of AOs centered on $\mathrm{M}$. We also note that only transitions with a small energy gap, $\varepsilon_{i}-\varepsilon_{a}$, can contribute efficiently to $\Delta \mathbf{g}^{\mathrm{SO} / \mathrm{OZ}}$. It will be shown (section Methods) that the coupling in the $\alpha$ and $\beta$ electron spin space in practice leads to negative $\left(-\Delta \mathbf{g}^{\alpha}\right)$ and positive $\left(+\Delta \mathbf{g}^{\beta}\right)$ contributions to the diagonal elements of the $\Delta \mathbf{g}^{\mathrm{SO} / \mathrm{OZ}}$ tensor, respectively. Its isotropic part then reads

$$
\Delta \mathrm{g}_{\mathrm{iso}}^{\mathrm{SO} / \mathrm{Oz}} \approx-\frac{1}{3}\left(\Delta \mathrm{g}_{x x}^{\alpha}+\Delta \mathrm{g}_{y y}^{\alpha}+\Delta \mathrm{g}_{z z}^{\alpha}\right)+\frac{1}{3}\left(\Delta \mathrm{g}_{x x}^{\beta}+\Delta \mathrm{g}_{y y}^{\beta}+\Delta \mathrm{g}_{z z}^{\beta}\right)
$$

where the quantities in parenthesis are positive. Therefore, any coupling involving the SOMO (occupied $\alpha$ spinor) results in shielding (negative $g$-shift, $g<2.0023$ ), whereas couplings involving the SUMO (vacant $\beta$ spinor) produce deshielding contributions (positive $g$-shift, $g>2.0023$ ). This is demonstrated for an open-shell square-plane model complex with a central metal atom $\mathrm{M}$ in Figure 2. For more details on Eq. (2) and Eq. (3), see section Methods.

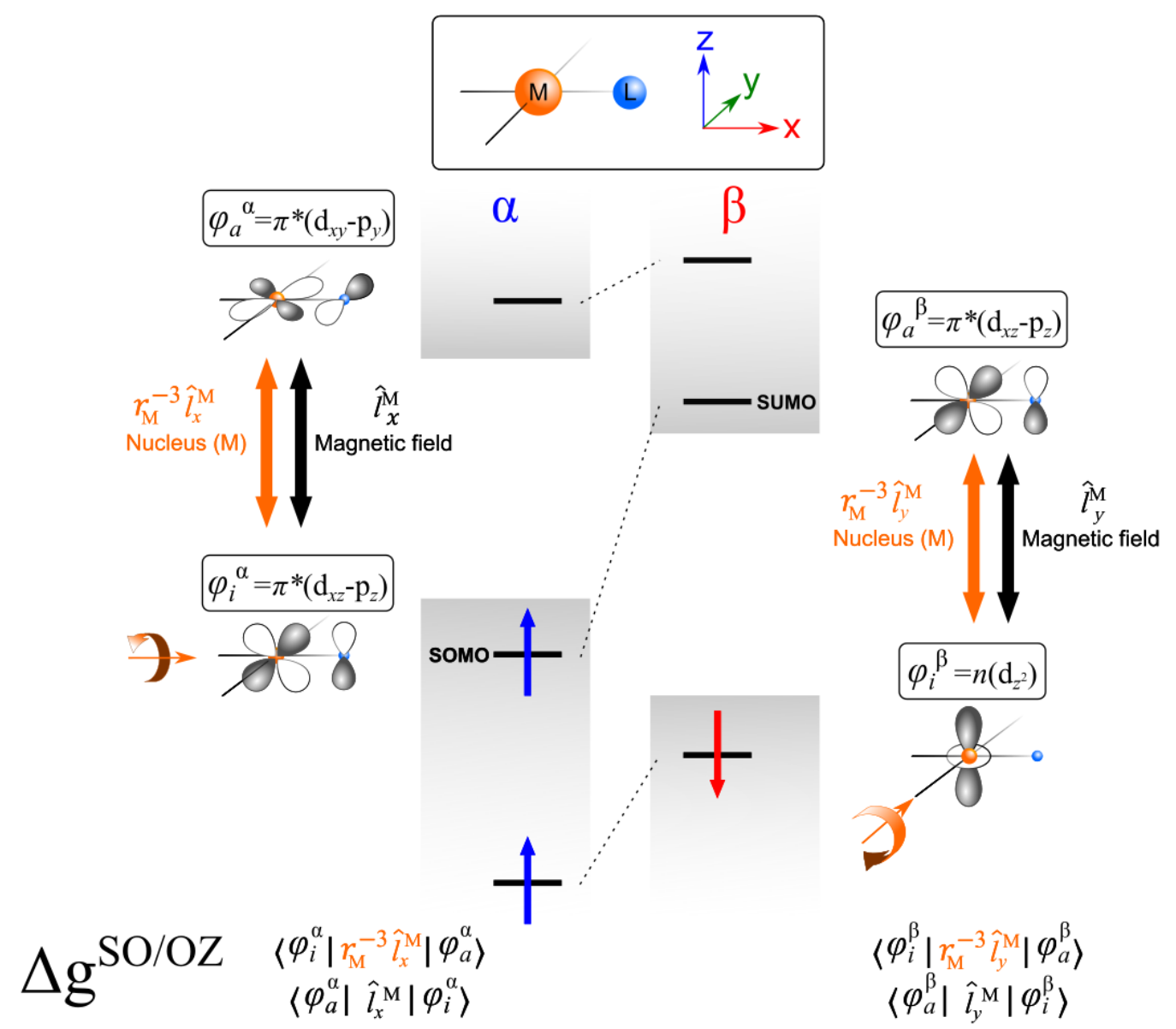

Figure 2. Electronic structure of a square-planar model complex with highlighted metal-ligand (M$\mathrm{L})$ bond and the selected $\mathrm{MO}^{\alpha} \leftrightarrow \mathrm{MO}^{\alpha *}$ and $\mathrm{MO}^{\beta} \leftrightarrow \mathrm{MO}^{\beta *}$ transitions relevant for the investigated $\mathrm{Ir}$ 
compounds, and the corresponding spin-orbit $\left(r_{M}^{-3} \hat{\boldsymbol{l}}^{M}\right)$ and magnetic field $\left(\hat{\boldsymbol{l}}^{M}\right)$ operators. For description and detailed discussion, see text.

Although the AOs of the ligand atom L are not important for explaining the $\mathrm{SO} / \mathrm{OZ}$ mechanism for the g-tensor of the metal-centered radical, they are included in Figure 2 to demonstrate the antibonding character of the g-active orbitals relative to the $\mathrm{M}-\mathrm{L}$ bond and for consistency with the hyperfine coupling tensor described in the next section. The SOMO is of $\pi^{*}\left(d_{\mathrm{xz}}-p_{\mathrm{z}}\right)$ nature and can be coupled to the vacant $\alpha$ spinor $\pi^{*}\left(d_{\mathrm{xy}}-p_{\mathrm{y}}\right)$ by the spin-orbit operator $\left(r_{M}^{-3} \hat{l}_{x}^{M}\right)$ when a magnetic field is applied in the $x$ direction $\left(\hat{l}_{x}^{M}\right)$, and leads to shielding of the $x$ component (negative contribution to $\left.\Delta \mathrm{g}_{x}\right)$. The SUMO also has $\pi^{*}\left(d_{\mathrm{xz}}-p_{\mathrm{z}}\right)$ character, but is coupled to the occupied $\beta$ spinor $n\left(d_{\mathrm{z}^{2}}\right)$ by the SO operator $\left(r_{M}^{-3} \hat{l}_{y}^{M}\right)$ when a magnetic field is applied in the $y$ direction $\left(\hat{l}_{y}^{M}\right)$ and induces deshielding of the $y$ component (positive contribution to $\Delta \mathrm{g}_{y}$ ). This simple analysis will be used to understand the specific $\mathrm{MO} \leftrightarrow \mathrm{MO} *$ transitions in complexes $\mathbf{1}$ and $\mathbf{3}$.

\section{Hyperfine Coupling Tensor - Nuclear-Spin-Electron-Orbit Operator}

In the framework of relativistic four-component theory used for the quantitative calculations in this work, the hyperfine coupling tensor of the ligand atom $\mathrm{L}\left(\mathbf{A}_{\mathrm{L}}\right)$ is obtained as the sum of the individual contributions of the Fermi-contact (FC), the spin-dipole (SD), the paramagnetic nuclear-spinelectron-orbit (PSO), and the pure relativistic (REL) terms ${ }^{26,27}$

$$
A_{\mathrm{L}}=\mathbf{A}_{\mathrm{L}}^{\mathrm{FC}}+\mathbf{A}_{\mathrm{L}}^{\mathrm{SD}}+\mathbf{A}_{\mathrm{L}}^{\mathrm{PSO}}+\mathbf{A}_{\mathrm{L}}^{\mathrm{REL}}
$$

As in the case of Eq. (1), the terms in Eq. (4) are calculated as inner products of the respective operators with the four-component wave function and thus contain both scalar-relativistic and spinorbit effects. The "through-bond" Fermi-contact contribution $\left(\mathbf{A}_{\mathrm{L}}^{\mathrm{FC}}\right)$ is generally very isotropic as it originates in the Fermi-contact interaction between the nuclear spin of atom $\mathrm{L}$ and the difference in $\alpha / \beta$ electron spin density in the core $s$-type orbitals at the nucleus. The FC contribution can thus be linked to the distribution of spin density in the system ${ }^{28-30}$ and analyzed in terms of spin delocalization and various spin-polarization mechanisms, ${ }^{30-32}$ see section Results and Discussion for more details. The spin-dipole term $\left(\mathrm{A}_{\mathrm{L}}^{\mathrm{SD}}\right)$ stems from the direct "through-space" interaction between the nuclear and electron magnetic moments and has traditionally been used as a source of information about the distance between a paramagnetic center and the NMR spectator atom in paramagnetic NMR spectroscopy. ${ }^{4,33}$ The leading-order correction to the paramagnetic nuclear-spin-electron-orbit term $\left(\mathbf{A}_{\mathrm{L}}^{\mathrm{PSO}}\right)$ originates in the interaction of the nuclear magnetic moment with the spin-orbit coupling, ${ }^{26,27}$ 
$\mathbf{A}_{\mathrm{L}}^{\mathrm{SO} / \mathrm{PSO}}$. This SO/PSO contribution is purely relativistic, analogous to the $\mathrm{SO} / \mathrm{OZ}$ contribution to $\mathbf{g}$

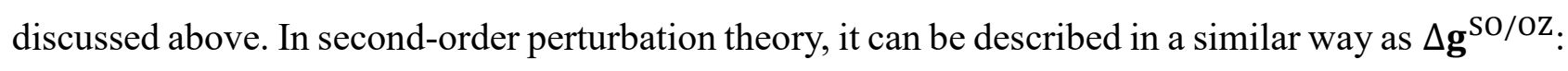

$$
\mathbf{A}_{\mathrm{L}}^{\mathrm{SO} / \mathrm{PSO}} \approx \sum_{i=1}^{\mathrm{occ}} \sum_{a=1}^{\mathrm{vac}}\left(\frac{\left\langle\varphi_{i}^{\alpha}\left|r_{M}^{-3} \hat{\boldsymbol{l}}^{M}\right| \varphi_{a}^{\alpha}\right\rangle\left\langle\varphi_{a}^{\alpha}\left|r_{L}^{-3} \hat{\boldsymbol{l}}^{L}\right| \varphi_{i}^{\alpha}\right\rangle}{\varepsilon_{i}^{\alpha}-\varepsilon_{a}^{\alpha}}-\frac{\left\langle\varphi_{i}^{\beta}\left|r_{M}^{-3} \hat{\boldsymbol{l}}^{M}\right| \varphi_{a}^{\beta}\right\rangle\left\langle\varphi_{a}^{\beta}\left|r_{L}^{-3} \hat{\boldsymbol{l}}^{L}\right| \varphi_{i}^{\beta}\right\rangle}{\varepsilon_{i}^{\beta}-\varepsilon_{a}^{\beta}}\right)
$$

In contrast to $\Delta \mathbf{g}^{\mathrm{SO} / \mathrm{OZ}}$, where only the metal-centered AOs govern the $\mathrm{SO} / \mathrm{OZ}$ mechanism via $\mathrm{SO}$ coupling, the AOs centered at the ligand atom L play an important role in the hyperfine SO/PSO mechanism. This is easily understandable because of the involvement of the nuclear magnetic moment of atom L via the $r_{L}^{-3} \hat{\boldsymbol{l}}^{L}$ operator in Eq. (5). Thus, the SO/PSO-active molecular orbitals must contain contributions from both the metal- and ligand-centered AOs. To keep our model simple, we demonstrate the SO/PSO mechanism (Figure 3) on the same set of molecular spinors as shown in Figure 2 for $\Delta \mathbf{g}^{\mathrm{SO} / \mathrm{OZ}}$.

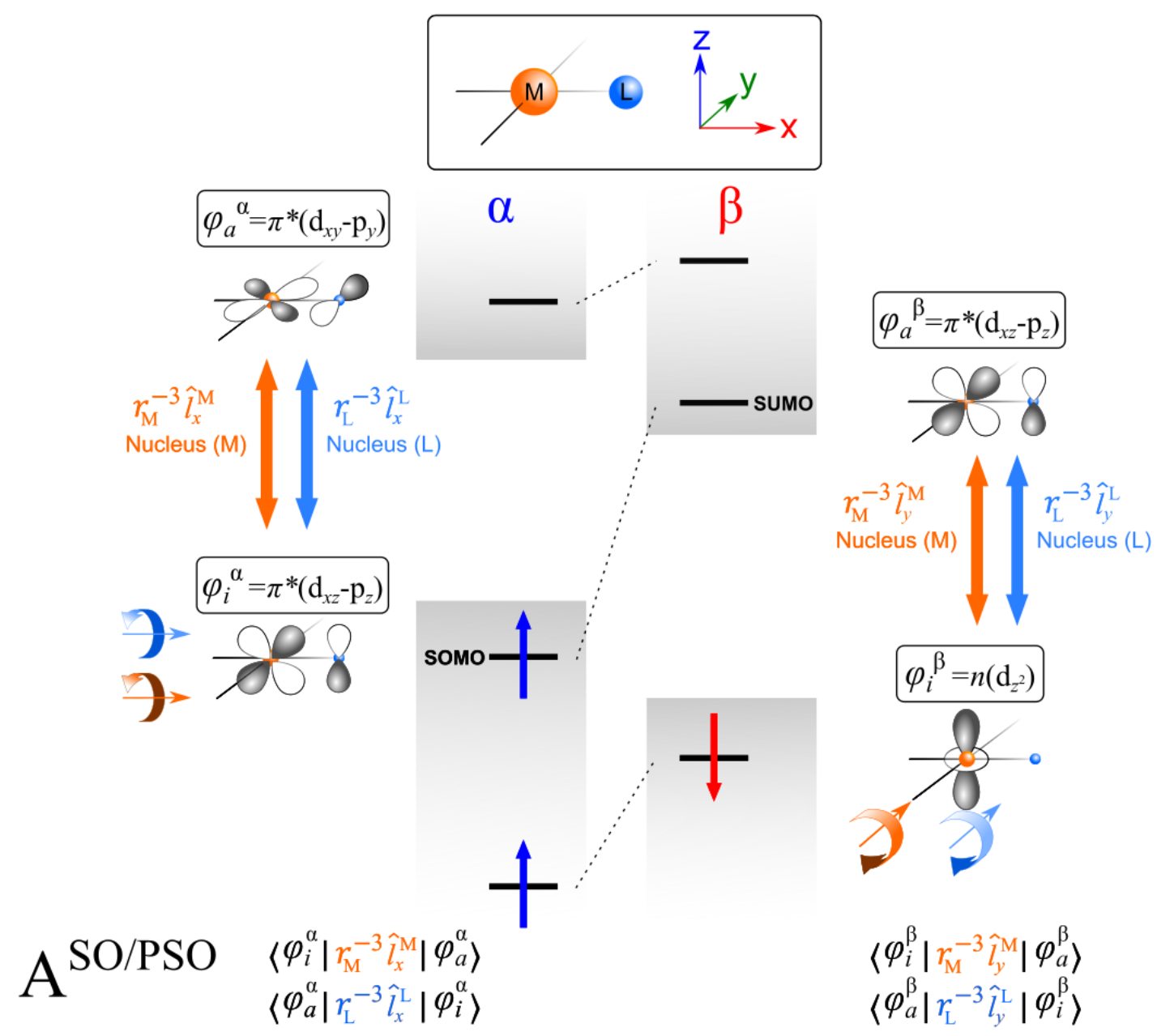

Figure 3. Schematic representation of the frontier molecular spinors for a model square-planar metal complex with the pertinent metal-ligand (M-L) bond, the selected $\mathrm{MO}^{\alpha} \leftrightarrow \mathrm{MO}^{\alpha *}$ and $\mathrm{MO}^{\beta} \leftrightarrow \mathrm{MO}^{\beta *}$ 
transitions, and the corresponding spin-orbit $\left(r_{M}^{-3} \hat{\boldsymbol{l}}^{M}\right)$ and nuclear-spin-electron-orbit $\left(r_{L}^{-3} \hat{\boldsymbol{l}}^{L}\right)$ operators. For description and detailed discussion, see text.

In analogy with the $\Delta \mathbf{g}^{\mathrm{SO} / \mathrm{OZ}}$ term, the SOMO of $\pi^{*}\left(d_{\mathrm{xz}}-p_{\mathrm{z}}\right)$ character is coupled to a vacant $\alpha$ spinor $\pi^{*}\left(d_{\mathrm{xy}}-p_{\mathrm{y}}\right)$. However, in contrast to $\Delta \mathbf{g}^{\mathrm{SO} / \mathrm{OZ}}$ where only the metal-centered AOs play an important role, contributions of the ligand-centered AOs in both the occupied and vacant spinors are now indispensable because of the role of the paramagnetic spin-orbit operator, $r_{L}^{-3} \hat{\boldsymbol{l}}^{L}$. Coupling in both the $\alpha$ and $\beta$ spaces can result in either positive or negative contributions to $\mathbf{A}^{\mathrm{SO} / \mathrm{PSO}}$, in contrast to $\Delta \mathbf{g}^{\mathrm{SO} / \mathrm{OZ}}$. The example given in the analysis of the $\mathbf{A}^{\text {SO/PSO }}$ mechanism for the $\beta$ space in Figure 3 , represented by $n\left(d_{\mathrm{z}^{2}}\right) \leftrightarrow \pi^{*}\left(d_{\mathrm{xz}}-p_{\mathrm{z}}\right)$, does not contribute to $\mathbf{A}^{\text {SO/PSO }}$ because there are no ligand AOs in the non-bonding spinor $n\left(d_{\mathrm{z}^{2}}\right)$. For the systems studied in this work, $\mathbf{A}^{\text {SO/PSO }}$ is instead governed by a different mechanism in $\beta$ space, as will be described in the section Results and Discussion.

\section{Hyperfine NMR Shift}

In the case of systems with doublet degeneracy of the ground state (a single unpaired electron), the total NMR chemical shift of ligand atom L is given by the sum of the orbital and hyperfine contributions:

$$
\delta_{\mathrm{L}}^{\mathrm{tot}}=\delta_{\mathrm{L}}^{\mathrm{orb}}+\delta_{\mathrm{L}}^{\mathrm{HF}}
$$

The calculation of the hyperfine NMR shift $\left(\delta_{\mathrm{L}}^{\mathrm{HF}}\right)$ can be directly related to the parameters of EPR spin Hamiltonian as shown for doublet systems by Moon and Patchkovskii ${ }^{5}$ and later extended to systems with an arbitrary spin degeneracy by Van den Heuvel and Soncini. ${ }^{34-38}$ The hyperfine shift can be constructed as the sum of the contributions derived from the isotropic and anisotropic parts of the $\mathbf{g}$ and A-tensors according to Eq. $(7)^{5,39}$ :

$$
\delta_{\mathrm{L}}^{\mathrm{HF}}=\delta_{\mathrm{L}}^{\mathrm{HFi}}+\delta_{\mathrm{L}}^{\mathrm{HFa}}=\frac{\mu_{e}}{4 \mathrm{kT} \gamma_{L}} g_{\text {iso }} A_{\text {iso }}(\mathrm{L})+\frac{\mu_{e}}{12 \mathrm{kT} \gamma_{L}} \operatorname{Tr}\left[\mathbf{g}_{\mathrm{ani}} \mathbf{A}_{\mathrm{ani}}(\mathrm{L})\right]
$$

where $\delta_{\mathrm{L}}^{\mathrm{HFi}}$ (derived from the isotropic $g$ and $A$ ) and $\delta_{\mathrm{L}}^{\mathrm{HFa}}$ (derived from the $\mathbf{g}$ and $\mathbf{A}$ anisotropy) contain the traditional contact and pseudocontact contributions, ${ }^{6,27,40}$ respectively. Note that according to the splitting of the hyperfine coupling tensor $\mathbf{A}_{\mathrm{L}}$ into FC, SD, and PSO terms, their individual contributions to the hyperfine NMR shift can be calculated and analyzed separately (for example, see ref. $^{27}$ ). 


\section{RESULTS AND DISCUSSION}

\section{Geometry Optimization and Experimental EPR and NMR Data}

To establish a quantitative link between the electronic spin structure and the relativistic magnetic resonance parameters, we performed a detailed analysis of the three square-planar iridium complexes $^{20,21}$ shown in Figure 1. The X-ray crystallographic structure available for compound $\mathbf{1}$ (CCDC$822068)^{20}$ was used as a starting point for the optimizing geometry. Molecular geometries for 2 and 3 were then constructed based on that of compound $\mathbf{1}$ by substituting $-\mathrm{N} 3$ or $\equiv \mathrm{N}$ for chlorine followed by full optimization of geometry (for additional geometry benchmarking calculations, see Tables S2S4 in Supporting Information). The most important metal-ligand interatomic distances obtained experimentally and calculated using the UPBE0 functional are summarized in Table 1.

Table 1. Metal-ligand (M-L) bond distances in compound 1 obtained from X-ray (CCDC No. 822068) and neutron (CCDC No. 822069) diffraction analyses ${ }^{20}$ and calculated by using DFT (UPBE0/def2ECP/def2-TZVPP and the COSMO model of toluene) in this work. Distances are given in pm. For Cartesian coordinates, see Supporting Information.

\begin{tabular}{cccc}
\hline Method & Ir-Cl & Ir-N & Ir-P \\
& & & \\
\hline Neutron diffraction $^{20}$ & 234.0 & 197.5 & 232.0 \\
X-ray diffraction $^{20}$ & 233.9 & 198.5 & 231.9 \\
DFT & 232.5 & 196.7 & 233.2 \\
\hline
\end{tabular}

The performance of the individual density functionals was compared by calculating $\mathrm{RMSD}_{\mathrm{M}-\mathrm{L}}$ for all of the metal-ligand bonds in compound 1 relative to the experimental values, see Tables S2-S4 in Supporting Information. The selected UPBE0 functional ${ }^{41}$ gives an RMSD value of approximately $1.3 \mathrm{pm}$ relative to the neutron-diffraction data ( $1.5 \mathrm{pm}$ relative to the $\mathrm{X}$-ray data), and very similar to those for UTPSSh ${ }^{42,43}$ and UMN12SX. ${ }^{44}$ However, as the PBE0 functional is used to calculate the response properties, we employed the PBE0-optimized geometries ${ }^{29,45}$ in the following analysis. The available experimental EPR and NMR data for compounds 1-3 are summarized in Table 2.

Table 2. Experimental values of the $g$-factor and principal components of $\mathbf{g}$, the $A$-constant and principal components of $\mathbf{A}$ (in MHz), and the ${ }^{1} \mathrm{H}$ NMR chemical shifts (in ppm) for compounds 1-3. 


\begin{tabular}{cccc}
\hline & Compound $\mathbf{1}^{20}$ & Compound $\mathbf{2}^{21}$ & Compound $\mathbf{3}^{21}$ \\
$g$ & $2.41^{\mathrm{a}}$ & $2.286^{\mathrm{b}}$ & $1.612^{\mathrm{b}}$ \\
$g_{11}$ & $-^{\mathrm{c}}$ & 3.091 & 1.885 \\
$g_{22}$ & $-^{\mathrm{c}}$ & 2.066 & 1.632 \\
$g_{33}$ & $-^{\mathrm{c}}$ & 1.700 & 1.320 \\
$A_{\mathrm{L}}$ & $-^{\mathrm{c}}$ & $-^{\mathrm{c}}$ & -8.3 \\
$A_{11}$ & $-^{\mathrm{c}}$ & $-^{\mathrm{c}}$ & $+63.5^{\mathrm{d}}$ \\
$A_{22}$ & $-^{\mathrm{c}}$ & $-^{\mathrm{c}}$ & $-26.5^{\mathrm{d}}$ \\
$A_{33}$ & $-^{\mathrm{c}}$ & $-^{\mathrm{c}}$ & $-62.0^{\mathrm{d}}$ \\
$\delta(\mathrm{H} 2)$ & $-6.8^{\mathrm{e}, \mathrm{f}}$ & $-5.6^{\mathrm{e}, \mathrm{f}}$ & $-49.2^{\mathrm{e}, \mathrm{g}}$ \\
$\delta(\mathrm{H} 3)$ & $-138.2^{\mathrm{e}, \mathrm{f}}$ & $-135.9^{\mathrm{e}, \mathrm{f}}$ & $-9.5^{\mathrm{e}, \mathrm{g}}$ \\
\hline
\end{tabular}

${ }^{\mathrm{a}}$ Measured in $\mathrm{Et}_{2} \mathrm{O}$-toluene glass at $86 \mathrm{~K} .{ }^{\mathrm{b}}$ Measured in toluene glass at $20 \mathrm{~K} .{ }^{\mathrm{c}}$ Not reported. ${ }^{\mathrm{d}}$ Obtained from X-band ENDOR measurement. ${ }^{\mathrm{e}}$ Not assigned in the original work - current assignment based on DFT calculations. ${ }^{\mathrm{f}}$ Measured in $\mathrm{C}_{6} \mathrm{D}_{6}\left(\delta_{\mathrm{H}}=7.16 \mathrm{ppm}\right.$ for residual $\left.\mathrm{C}_{6} \mathrm{D}_{5} \mathrm{H}\right)$ at $293 \mathrm{~K} .{ }^{\mathrm{g}}$ Measured in THF- $d_{8}\left(\delta_{\mathrm{H}}=3.58 \mathrm{ppm}\right.$ for residual THF- $\left.d_{7}\right)$ at $293 \mathrm{~K}$.

\section{Calculation of EPR and NMR Parameters}

Effects of Solvent and Relativity on EPR Parameters.

We performed a systematic study of the effects of the choice of exchange-correlation (XC) functional (particularly the user-defined amount of exact exchange in PBE0) and basis set on the calculated EPR data, see Figures S1 and S2 in Supporting Information. Following our recent benchmark studies, ${ }^{30,46}$ we employed the standard PBE0 functional with a basis set of double- $\zeta(2-\zeta)$ quality for the production calculations. We compare our DFT results with available experimental data for selected magneticresonance parameters of compounds $\mathbf{1}$ and $\mathbf{3}$ in Table 3.

Table 3. Selected experimental and DFT (DKS/PBE0/vdz/upcJ-1/PCM) values of $\mathbf{g}, \mathbf{A}(\mathrm{L} 4)$, and total $\delta$ for $\mathrm{H} 2$ and $\mathrm{H} 3$ in compounds $\mathbf{1}$ and $\mathbf{3}$ (geometries optimized in solvent). For 1: EPR in Et $2 \mathrm{O}$-toluene $(1: 1)$ and NMR in benzene; for 3: EPR in toluene and NMR in tetrahydrofuran, see Table 2.

\begin{tabular}{ccccc}
\hline & \multicolumn{2}{c}{$\mathbf{3}$} \\
\hline & Exp & Calc & Exp & Calc \\
$g$ & 2.41 & 2.237 & 1.612 & 1.729 \\
$g_{11}$ & $-^{\mathrm{a}}$ & 3.482 & 1.885 & 1.976 \\
$g_{22}$ & $-^{\mathrm{a}}$ & 1.860 & 1.632 & 1.740
\end{tabular}




$\begin{array}{ccccc}g_{33} & -^{\mathrm{a}} & 1.369 & 1.320 & 1.471 \\ A_{\mathrm{L}} & -^{\mathrm{a}} & +12.2 & -8.3 & -8.5 \\ A_{11} & -^{\mathrm{a}} & +18.6 & +63.5 & +66.1 \\ A_{22} & -^{\mathrm{a}} & +11.5 & -26.5 & -28.1 \\ A_{33} & -^{\mathrm{a}} & +6.5 & -62.0 & -63.4 \\ \delta(\mathrm{H} 2) & -6.8^{\mathrm{b}} & +9.5^{\mathrm{c}} & -49.2^{\mathrm{b}} & -57.8^{\mathrm{c}} \\ \delta(\mathrm{H} 3) & -138.2^{\mathrm{b}} & -131.8^{\mathrm{c}} & -9.5^{\mathrm{b}} & -9.6^{\mathrm{c}}\end{array}$

${ }^{\mathrm{a}}$ Not reported. ${ }^{\mathrm{b}}$ Not assigned. ${ }^{\mathrm{c}}$ Calculated as the sum of the orbital $\left(\delta^{\text {orb }}\right)$ and hyperfine $\left(\delta^{\mathrm{HF}}\right)$ shifts, see Eq. (6) and Table S5 in Supporting Information.

It has been demonstrated that EPR and NMR parameters are very sensitive to environmental (including solvent) ${ }^{29,47}$ and relativistic effects. ${ }^{5}$ Because only limited experimental EPR and NMR data for compounds 1-2 were available, we selected compound $\mathbf{3}$ for the methodological testing as it has the most complete set of data (Table 3$)$ and the most challenging bonding situation. Solvent $\left(\Delta_{\text {sol }}\right)$ and spin-orbit $\left(\Delta_{\mathrm{SO}}\right)$ corrections to the $g$-factor and ligand HF coupling constants $(A)$, and their effects on the hyperfine ${ }^{1} \mathrm{H}$ and ${ }^{13} \mathrm{C}$ NMR shifts were analyzed using the DKS approach, and the results are summarized in Table 4.

Table 4. Values of $g_{\text {iso }}$ and $A_{\text {iso }}$ constants (in $\mathrm{MHz}$ ) for selected atoms in compound 3 (geometry optimized in toluene) obtained using DFT. ${ }^{\text {a }}$ The values were calculated at the scalar-relativistic DKS (sc-DKS) or four-component DKS (DKS) level in vacuum or using the polarizable-continuum model for the solvent (PCM, toluene) with spin-orbit $\left(\Delta_{\mathrm{SO}}\right)$ and solvent $\left(\Delta_{\text {sol }}\right)$ corrections shown separately.

\begin{tabular}{cccccc}
\hline & $\mathrm{sc}-\mathrm{DKS}^{\mathrm{b}}$ & $\Delta_{\text {so }}$ & $\mathrm{DKS}^{\mathrm{b}}$ & $\Delta_{\text {sol }}$ & $\mathrm{DKS}^{\mathrm{c}}$ \\
$g$ & $2.002^{\mathrm{d}}$ & -0.323 & 1.679 & +0.050 & 1.729 \\
$A(\mathrm{~N} 1)$ & -5.53 & +0.56 & -4.97 & +0.29 & -4.68 \\
$A(\mathrm{~N} 4)$ & $\mathbf{+ 8 . 9 2}$ & $\mathbf{- 1 9 . 0 8}$ & $\mathbf{- 1 0 . 1 6}$ & $\mathbf{+ 1 . 6 9}$ & $\mathbf{- 8 . 4 7}$ \\
$A(\mathrm{C} 2)$ & +2.07 & +0.04 & +2.11 & -0.42 & +1.69 \\
$A(\mathrm{C} 3)$ & -0.45 & +0.01 & -0.44 & +0.22 & -0.22 \\
$A(\mathrm{H} 2)$ & -3.06 & +0.17 & -2.89 & +0.11 & -2.78 \\
$A(\mathrm{H} 3)$ & -0.32 & -0.06 & -0.38 & -0.16 & -0.54 \\
\hline
\end{tabular}

${ }^{\mathrm{a}} \mathrm{PBE} 0 / \mathrm{vdz} / \mathrm{upcJ}-1 .{ }^{\mathrm{b}}$ Calculated in vacuum. ${ }^{\mathrm{c}}$ Calculated by using the PCM model of solvent (toluene) used in the experimental measurement (see Table 2). ${ }^{\mathrm{d}}$ This corresponds to the free-electron value, $g_{\mathrm{e}}$. For more data, see Table S6 in Supporting Information. 
As expected, the solvent effects are as important, or even more important, than the SO effects for the ligand carbon and hydrogen atoms most distant from the iridium center. This is because these atoms are located more on the periphery and are therefore more exposed to the solvent, in contrast to the central iridium core which is somewhat screened from the solvent environment by bulky tert-butyl groups. The SO coupling induces a $g$-shift of about -323 ppt and dramatically affects the $A$-coupling for atom N4 which is bonded directly to iridium. Here the SO effect is $-19.1 \mathrm{MHz}$, reversing the sign of $A(\mathrm{~N} 4)$ from $+8.9 \mathrm{MHz}$ to $-10.2 \mathrm{MHz}$. The solvent slightly counter-balances the $A$-value by +1.7 $\mathrm{MHz}$, resulting in a final value of $-8.5 \mathrm{MHz}$, in excellent agreement with the experimental value of 8.3 MHz. The SO effects are discussed in more detail in the following sections.

\section{Analysis of Spin-Orbit Effects on $\mathbf{g}$ and $\boldsymbol{A}$}

As demonstrated in the previous section, the fully-relativistic DKS method with the hybrid PBE0 functional and the $2-\zeta$ quality basis set (vdz/upcJ-1/PCM) reproduces the experimental EPR parameters very well, and suggests that a detailed analysis of $\mathbf{g}$ and $\mathbf{A}$ using this methodology may help unravel the electronic structure of these molecules. As solvent effects do not change the qualitative trends, the following calculations have been performed in vacuum.

To assess the role of greater-than-linear spin-orbit effects on the calculated $\mathbf{g}$ and $\mathbf{A}$ values, we performed several calculations with a scaled weight of the SO operator (see section Methods). The resulting profiles of the $g$ and $A(\mathrm{~L})$ parameters for compounds $\mathbf{1}$ and $\mathbf{3}$ are shown in Figure 4. 

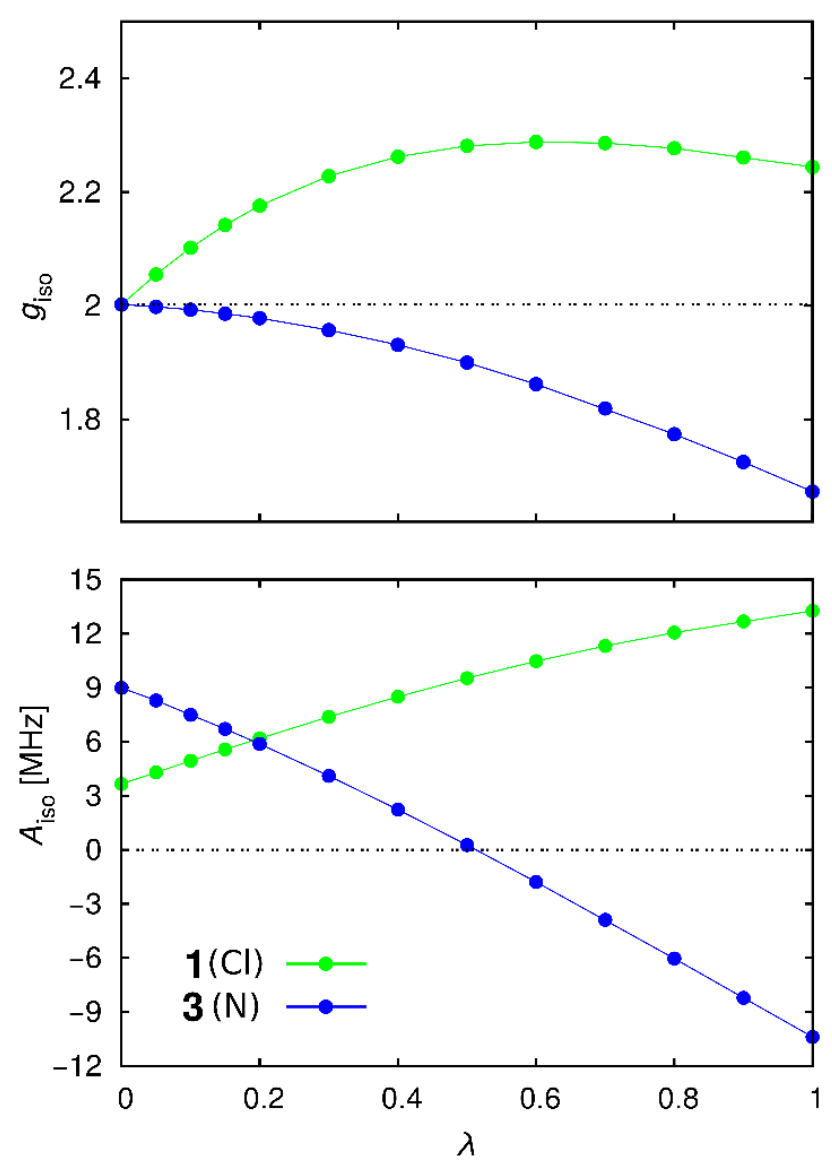

Figure 4. Scaling the SO contribution in the DKS calculation of $g$ (top) and $A_{\mathrm{L}}$ (bottom, in $\mathrm{MHz}$ ) for compounds 1 and 3. Scaling factor $\lambda=0$ corresponds to the scalar-relativistic limit, whereas $\lambda=1$ stands for the fully-relativistic treatment. For analysis of the principal components of $\mathbf{g}$ and $\mathbf{A}$, and the "speed of light scaling", see Figures S3 and S4 in Supporting Information.

These profiles demonstrate that the dependence for $A$ is approximately linear and is only marginally affected by higher-order spin-orbit effects. The situation is slightly more complicated for $g$, where the profiles deviate notably from linearity, particularly for compound $\mathbf{1}$. However, the sign differences in both EPR parameters ( $\Delta g$ and $A$ ) between compounds $\mathbf{1}$ (positive) and $\mathbf{3}$ (negative) can be explained sufficiently by the linear spin-orbit effects and, therefore, should be transparent from the corresponding analysis based on second-order perturbation theory (PT2). It would be interesting to analyze and understand the quadratic spin-orbit effects, but this requires the implementation of thirdorder perturbation theory (PT3), which is beyond the scope of this work.

In the next step, the hyperfine coupling constants calculated using PBE0/2- $\zeta$ in vacuum were analyzed in terms of the Fermi-contact (FC), paramagnetic spin-orbit (PSO), and spin-dipole (SD) contributions. $^{27,29}$ The results are summarized in Table 5. Significant differences in the individual terms between compounds $\mathbf{1}$ and $\mathbf{3}$, including the opposite signs of $A^{\mathrm{PSO}}$ for ligand atom L4, opposite signs of $A^{\mathrm{FC}}$ for $\mathrm{C} 2$ and $\mathrm{C} 3$, and large hyperfine NMR shifts for $\mathrm{H} 2$ in $\mathbf{3}$ vs $\mathrm{H} 3$ in $\mathbf{1}$ are highlighted in 
bold. These differences are tightly connected to the electronic spin structure around the central iridium atom and the character of the metal-ligand bond.

Table 5. The hyperfine coupling constants $\left(A_{\text {iso }}\right.$ in $\left.\mathrm{MHz}\right)$ - with their separate Fermi-contact (FC), paramagnetic spin-orbit (PSO), and spin-dipole (SD) contributions - and hyperfine NMR shifts $\left(\delta^{\mathrm{HF}}\right.$ in $\mathrm{ppm})^{\mathrm{a}}$ calculated for compounds $\mathbf{1}$ and $\mathbf{3}$ (geometries optimized in vacuum) using the DKS approach with the PBE0 functional and the $2-\zeta$ basis set in vacuum.

\begin{tabular}{ccccccc}
\hline Compound & Atom & $A_{\text {iso }}$ & $A^{\mathrm{FC}}$ & $A^{\mathrm{PSO}}$ & $A^{\mathrm{SD}}$ & $\delta^{\mathrm{HF}}$ \\
& $\mathrm{C} 14$ & +13.27 & +2.76 & $+\mathbf{1 1 . 3 1}$ & -0.73 & +3414 \\
& $\mathrm{P}$ & -13.48 & -15.68 & +1.97 & -0.18 & -1012 \\
& $\mathrm{~N} 1$ & -0.66 & -1.16 & +0.52 & -0.03 & -682 \\
& $\mathrm{C} 2$ & $\mathbf{- 6 . 1 0}$ & -5.82 & -0.30 & 0.001 & -686 \\
& $\mathrm{C} 3$ & $\mathbf{+ 4 . 4 7}$ & +4.54 & -0.12 & +0.05 & +311 \\
& $\mathrm{H} 2$ & -0.23 & -0.08 & -0.08 & -0.06 & -10.4 \\
& $\mathrm{H} 3$ & $\mathbf{- 4 . 3 2}$ & $\mathbf{- 4 . 4 4}$ & +0.04 & +0.08 & $\mathbf{- 1 2 0 . 0}$ \\
& $\mathrm{N} 4$ & -10.39 & +7.95 & $-\mathbf{1 6 . 8 4}$ & -1.47 & -2496 \\
& $\mathrm{P}$ & $\mathbf{- 3 6 . 5 0}$ & -34.94 & -3.40 & +0.91 & -2114 \\
& $\mathrm{~N} 1$ & -4.94 & -5.27 & +0.37 & -0.07 & -1460 \\
& $\mathrm{C} 2$ & $\mathbf{+ 2 . 0 9}$ & +1.84 & +0.23 & +0.02 & +178 \\
& $\mathrm{C} 3$ & $\mathbf{- 0 . 4 4}$ & -0.31 & -0.15 & +0.01 & -39 \\
& $\mathrm{H} 2$ & $\mathbf{- 2 . 9 0}$ & $\mathbf{- 2 . 7 8}$ & -0.15 & +0.03 & $\mathbf{- 6 9 . 1}$ \\
& $\mathrm{H} 3$ & -0.36 & -0.34 & -0.06 & +0.05 & -7.9 \\
\hline
\end{tabular}

${ }^{\text {a }}$ Calculated for a temperature of $293 \mathrm{~K}$.

\section{Comparison of the Electronic Structures of Compounds 1 and 3 and Interpretation of the Differences in EPR and NMR Data}

Compounds 1 and $\mathbf{3}$ differ in the ligand atom L (chlorine or nitrogen) and display major differences in their EPR and NMR parameters. What are the origins of these differences? The energy-level diagram of the significant Kohn-Sham molecular spin-orbitals (MSOs) with contributions from the iridium 5d AOs in compounds $\mathbf{1}$ and $\mathbf{3}$ is shown in Figure 5. Both the $\alpha$ and $\beta$ MSOs with contributions of Ir $d_{\mathrm{yz}}$ and $d_{\mathrm{z}^{2}}$ are only marginally affected by the substitution of $\mathrm{N}$ for $\mathrm{Cl}$, as they are oriented in the plane perpendicular to the $\mathrm{Ir}-\mathrm{L}$ bond. However, the formation of a strong iridium-nitrogen triple bond (not shown in Figure 5) destabilizes the antibonding orbitals (both $\alpha$ and $\beta$ spinors) that involve 
Ir $d_{\mathrm{xz}}\left(\alpha \mathrm{SOMO}\right.$ and $\beta$ SUMO) and in particular $d_{\mathrm{xy}}$. This energy change shifts the $d_{\mathrm{xy}}$-based MSOs from the occupied (1) to the vacant (3) spinor space (the importance of which for $\mathbf{g}$ and $\mathbf{A}$ will be shown). The energy destabilization of the above-mentioned $\mathrm{Ir}-\mathrm{L}$ antibonding MSOs can intuitively be related to the change in the formal oxidation state of the Ir atom from + II in $\mathbf{1}$ to + IV in $\mathbf{3}$, by removing two electrons from the iridium center.

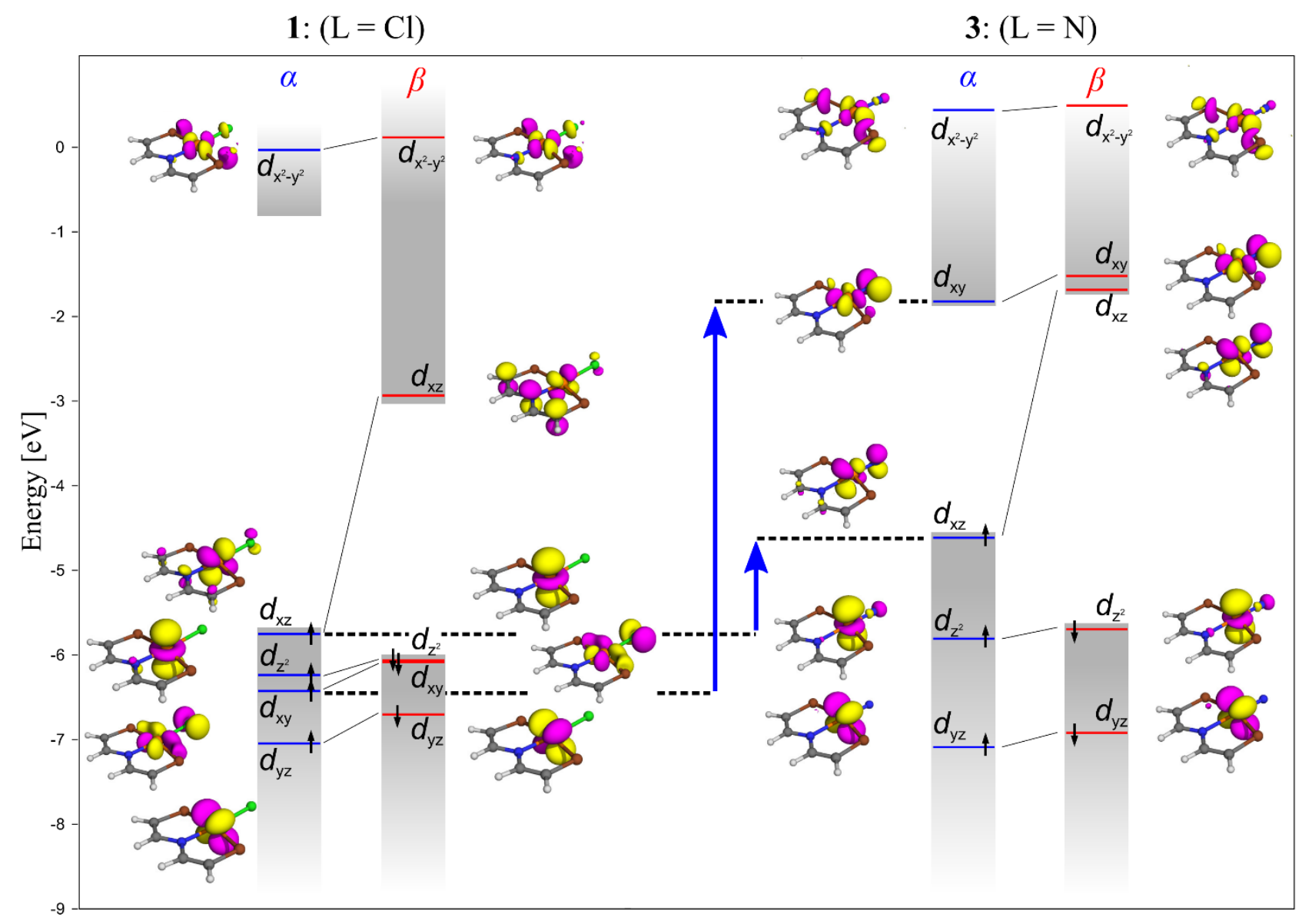

Figure 5. Schematic energy-level diagram $(\mathrm{eV})$ of the frontier metal-centered molecular spinors (MSOs) for compounds $\mathbf{1}$ (left) and $\mathbf{3}$ (right). Frontier ligand-centered MSOs are omitted for clarity. Note the significant destabilization (delineated by the blue arrow) of the $d_{\mathrm{xz}^{-}}$and particularly the $d_{\mathrm{xy}}-$ type MSOs in compound $\mathbf{3}$ compared to those in $\mathbf{1}$.

The SOMO (Ir $d_{\mathrm{xz}}$-based) has different admixtures of atomic orbitals from the two trans ligands in 1 and $\mathbf{3}$ as shown in Figure 6a. This is related to the structural trans effect where the two ligand atoms share a single orbital of the central metal atom. ${ }^{48-51}$ Therefore, the chlorine atom $\mathrm{Cl} 4$ that is weakly bonded to the central iridium allows for a relatively strong trans $\mathrm{Ir}-\mathrm{N} 1$ bond in compound $\mathbf{1}$. In contrast, the triple-bonded nitrogen ligand atom (N4) in compound $\mathbf{3}$ prevents the formation of a strong $\mathrm{Ir}-\mathrm{N} 1$ bond. These differences in the magnitudes of the $\mathrm{Ir} \leftrightarrow \mathrm{L}$ electron sharing (bond covalence $^{52}$ are clearly reflected in the delocalization indices (DI) $)^{53,54}$ of QTAIM, see Figure $6 \mathrm{~b}$. The 
$\mathrm{DI}(\mathrm{N} 1 \leftrightarrow \mathrm{Ir})$ values are 1.00 and 0.58 au for compound $\mathbf{1}$ and $\mathbf{3}$, respectively. Therefore, the SOMO (Figure 6a) is notably more delocalized towards the $\mathrm{N}-\mathrm{C}=\mathrm{C}$ fragment in $\mathbf{1}$, whereas the presence of the $\mathrm{Ir}=\mathrm{N}$ triple bond in $\mathbf{3}$ allows for an efficient spin sharing between Ir and N4. We will now investigate how these different bonding situations are reflected in the magnetic resonance parameters.

a)
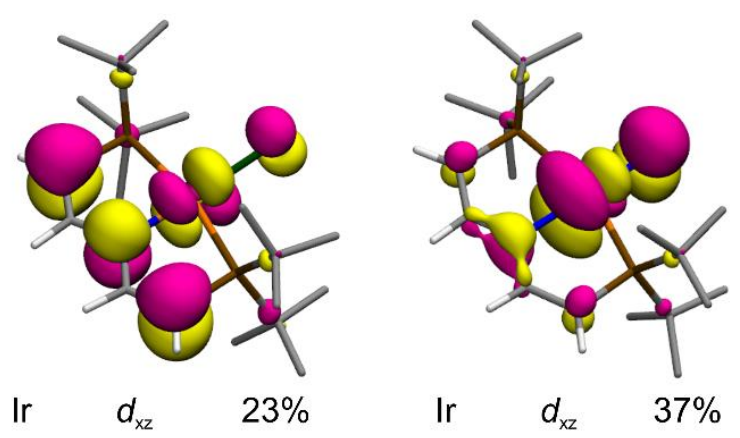

$\mathrm{Cl} 4 \quad p_{z} \quad 6 \%$

C3 $p_{z} \quad 20 \%$

C3' $p^{\prime} \quad 20 \%$

N1 $\quad p_{z} \quad 21 \%$

$\begin{array}{llr}\text { Ir } & d_{x z} & 37 \% \\ \text { N4 } & p_{z} & 33 \% \\ \text { C3 } & p_{z} & 4 \% \\ \text { C3' } & p_{z} & 4 \% \\ \text { N1 } & p_{z} & 3 \%\end{array}$

b)
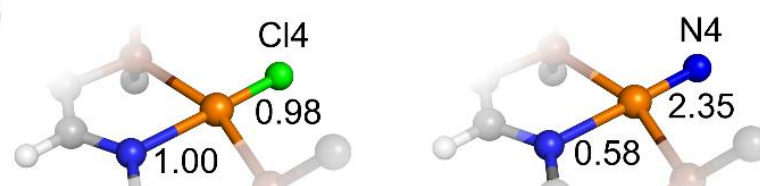

Figure 6. a) Visualization of SOMOs ( $\alpha$ spinor, calculated by sc-DKH2/PBE0/upcJ-1/vacuum) with different spin delocalization (Mulliken analysis) ${ }^{55}$ by contributing ligand atomic orbitals (AOs) in 1 and 3. b) Central fragment N1-Ir-L4 in compounds 1 and $\mathbf{3}$ with QTAIM electron-delocalization indices (DIs) for N1-Ir and Ir-L bonds.

\section{“Long-Range” Fermi-Contact (FC) Contributions to Hyperfine ${ }^{1} \mathrm{H}$ and ${ }^{13} \mathrm{C}$ NMR Shifts}

The more covalent Ir-N1 bond in compound $\mathbf{1}$ compared to that in $\mathbf{3}$ enables significant involvement of the $\mathrm{N} 1$ and $\mathrm{C} 2-\mathrm{C} 3 \mathrm{AOs}$ in the SOMO (Figure 6a) and consequently a higher degree of spin delocalization on the $\mathrm{N}-\mathrm{C}=\mathrm{C}$ fragment via the $\pi$-space (cf. the magnitudes of $A_{\text {iso }}(\mathrm{C} 2)$ and $A_{\text {iso }}(\mathrm{C} 3$ ) in Table 5). The opposite signs of $A_{\mathrm{iso}}(\mathrm{C} 2)$ and $A_{\mathrm{iso}}(\mathrm{C} 3)$ in compounds $\mathbf{1}$ and $\mathbf{3}$ originate from the opposite signs of the Fermi-contact contributions (Table 5) and are directly linked to the distribution of spin density in the systems, see Figure 7. This can be rationalized as follows. Compound 1 has a covalent Ir-N1 bond where the $\alpha$-spin density from the Ir atom is directly delocalized to the $\pi$-space of N1 (blue lobe). In contrast, the weak Ir-N1 bond in compound 3 quenches the spin delocalization to $\mathrm{N} 1$ and the spin density is propagated through spin polarization, inducing an overabundance of $\beta$ spin in the $\pi$-space of N1 (red lobe), see Figure 7. This opposite spin polarization in the $\pi(\mathrm{N} 1)$-space 
is further propagated to the $\mathrm{C}=\mathrm{C}$ fragment in subsequent polarization steps. ${ }^{27,30,32,56,57}$ Thus, the predominance of $\beta$-density (red) and $\alpha$-density (blue) is clearly identified in the $\pi$-space of C2 in 1 and 3, respectively. This inverse $\pi(\mathrm{C} 2)$ spin polarization (Table 6) is further propagated in an alternating way to carbon atom $\mathrm{C} 3$ in $\mathbf{1}$ and becomes rather marginal for $\mathrm{C} 3$ in $\mathbf{3}$ because of the much less effective polarization pathway originating in the $\pi$-space of $\mathrm{N} 1$ and a possible additional counterbalancing pathway involving the phosphorus atom (cf. $A_{\text {iso }}$ values in Table 6). In accordance with the $\beta$ spin density on the hydrogen atoms in Figure 7, a significantly negative $A_{\mathrm{iso}}^{\mathrm{FC}}$ is observed for $\mathrm{H} 3$ in $\mathbf{1}$ and $\mathrm{H} 2$ in $\mathbf{3}$. Clearly, this analysis substantiates our current assignment of the originally unassigned ${ }^{1} \mathrm{H}$ NMR resonances of $\mathrm{H} 2$ and $\mathrm{H} 3$ as summarized in Tables 2 and 3.
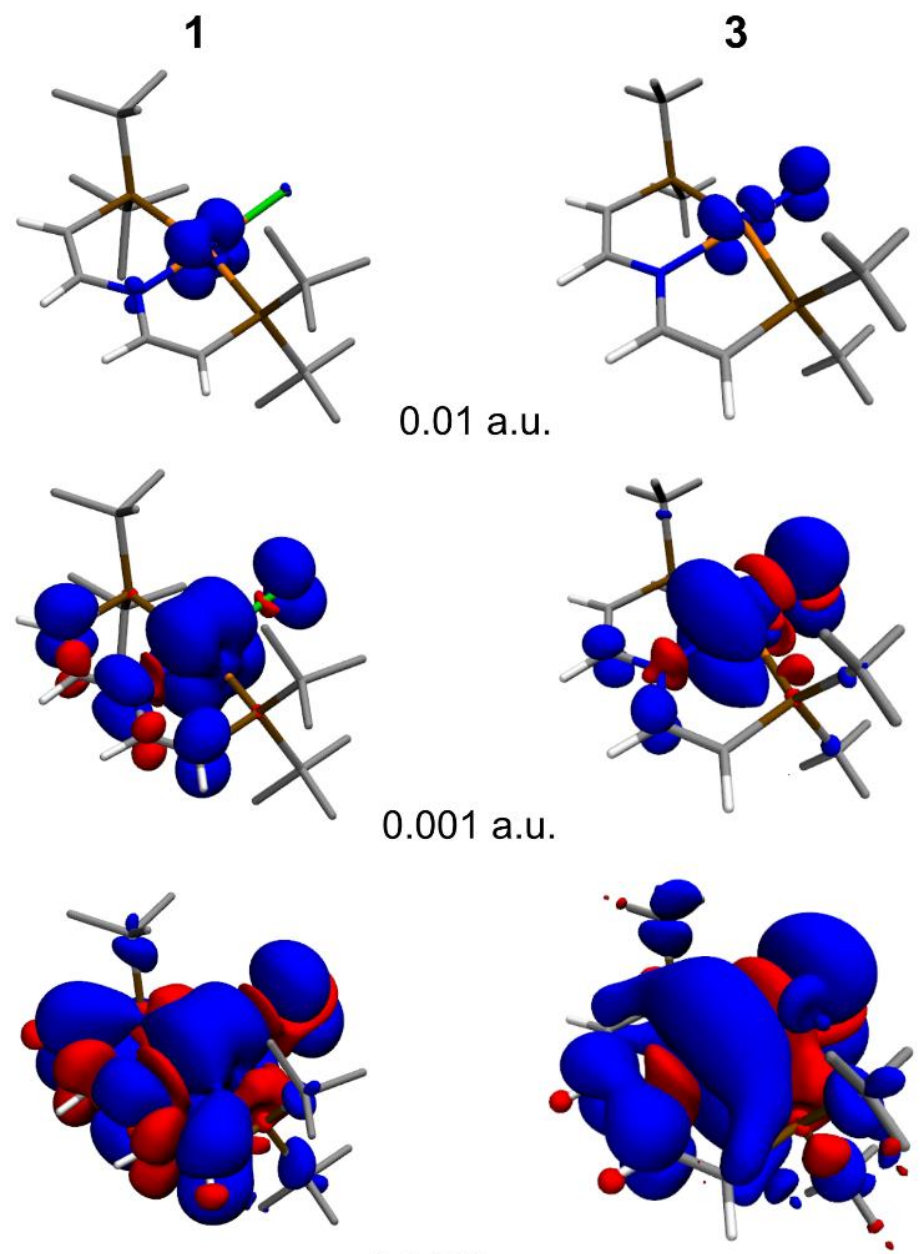

0.0002 a.u.

Figure 7. Visualization of the $z$-component of the $z$-magnetized spin density at 0.01 a.u. (top), 0.001 a.u. (middle), and 0.0002 a.u. (bottom) for compounds $\mathbf{1}$ (left) and $\mathbf{3}$ (right) calculated at the fourcomponent DKS level (PBE0/vdz/upcJ-1) in vacuum. The predominance of $\alpha$ and $\beta$ spin density is shown in blue and red, respectively. For $\mathrm{C} 2$ and $\mathrm{C} 3$ atomic spin populations, see Table 6.

Table 6. Fermi-contact contribution $\left(A^{\mathrm{FC}}\right)$ to the hyperfine coupling constant of atoms $\mathrm{C} 2$ and $\mathrm{C} 3$ calculated at the DKS and sc-DKS level in vacuum in ReSpect, and spin population on the $2 p_{\pi}$ atomic 
orbital of carbon atoms $\mathrm{C} 2$ and $\mathrm{C} 3$ in compounds $\mathbf{1}$ and $\mathbf{3}$ calculated at the PBE0/ECP/def2TZVPP/vacuum level in Gaussian $16^{58}$

\begin{tabular}{ccccr}
\hline Compound & Atom & $A^{\mathrm{FC}}(\mathrm{DKS})$ & $A^{\mathrm{FC}}(\mathrm{sc}-\mathrm{DKS})$ & $2 p_{\pi}$ \\
$\mathbf{1}$ & $\mathrm{C} 2$ & -5.82 & -8.15 & -0.028 \\
& $\mathrm{C} 3$ & +4.54 & +6.34 & +0.074 \\
\multirow{3}{*}{3} & $\mathrm{C} 2$ & +1.84 & +2.06 & +0.020 \\
& $\mathrm{C} 3$ & -0.31 & -0.47 & -0.001 \\
\hline
\end{tabular}

Electronic $\mathbf{g}$-tensor: Origin of the Sign of $\Delta g$ in Compounds $\mathbf{1}$ and $\mathbf{3}$

The isotropic $g$-factor ( $g_{\text {iso }}$ in vacuum, 2.244 for $\mathbf{1}, 1.673$ for $\mathbf{3}$ ) and the different components of $\mathbf{g}$, Figure 8, reflect the different bonding to ligands L4 and N1 and the accompanying difference in spin delocalization in compounds 1 (total Ir spin population 0.79 at sc-DKH2/PBE0/vdz/upcJ-1 level in vacuum) and 3 (0.41). Note in particular the large values for $g_{\mathrm{y}}$ (green, $+1472 \mathrm{ppt}$ ) and $g_{\mathrm{x}}(\mathrm{red},-599$ ppt) in compounds $\mathbf{1}$ and $\mathbf{3}$, respectively. In order to understand these differences in $\Delta \mathbf{g}$ between compounds $\mathbf{1}$ and 3, we again turn to second-order perturbation theory (PT2), which is justified based on the good agreement between the results we obtained at the full DKS level (PBE0 and PBE functionals) and using sensible approximations in the PT2 theory, see Tables S7 and S8 in Supporting Information.

a)

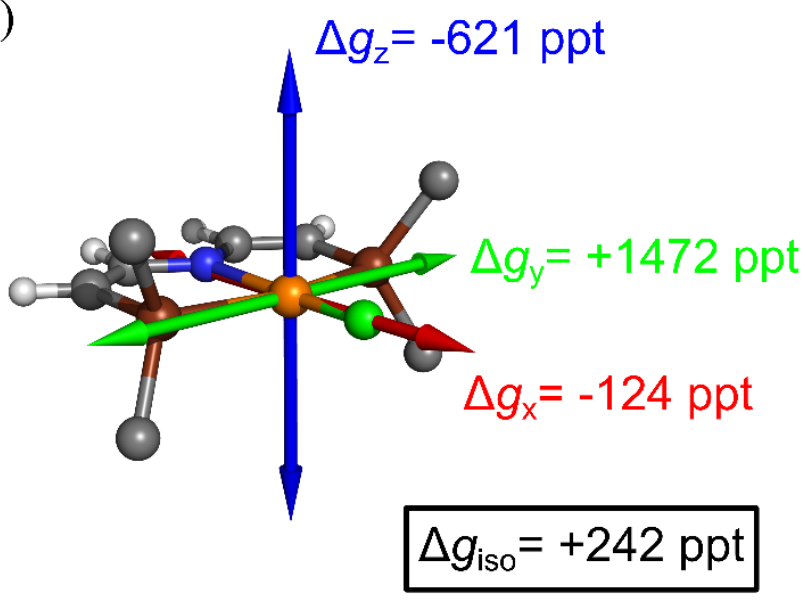

b)

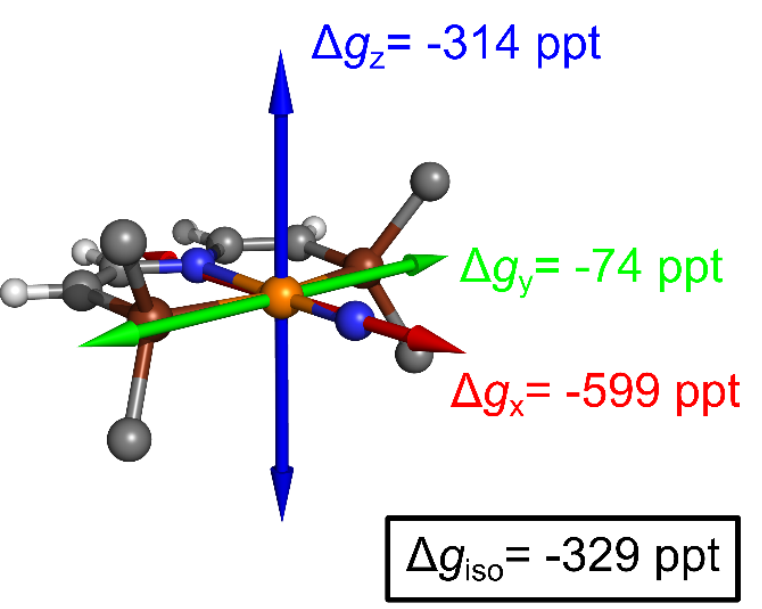

Figure 8. Visualization of components of the electronic $\mathbf{g}$-shift tensor $(\Delta \mathbf{g})$ in the molecular coordinate system for a) compound $\mathbf{1}$ and b) compound $\mathbf{3}$ calculated by using the DKS approach (PBE0/vdz/upcJ-1/vacuum). Note particularly the large positive $\Delta g_{\mathrm{y}}$ (green) in $\mathbf{1}$ and the large negative $\Delta g_{\mathrm{x}}$ (red) in $\mathbf{3}$. Because of the somewhat more localized spin density at iridium atom in $\mathbf{1}$ (total Ir spin population 0.79 calculated by sc-DKH2/PBE0/vdz/upcJ-1/vacuum) comparing to 3 (total Ir spin population 0.41), its $\mathbf{g}$ span $\left(g_{\mathrm{y}}-g_{\mathrm{z}}=2093 \mathrm{ppt}\right)$ is notably larger. 
From the MSO diagram in Figure 5, it is clear that the magnetic couplings between the occupied and vacant $\alpha$ spinors would not be very efficient in compound 1 due to the large $\mathrm{MO} \leftrightarrow \mathrm{MO} *$ energy gap $(>5 \mathrm{eV})$, and particularly the absence of a vacant $\alpha \mathrm{MSO}$ of the appropriate composition and symmetry, as this would require the coupling of the SOMO ( $\alpha d_{\mathrm{xz}}$-based) to the Ir $d_{\mathrm{xy}}$ - or $d_{\mathrm{yz}}$-based MSOs, which are hardly available in the vacant $\alpha$ space as the LUMO is mostly centered on the ligand. Therefore, coupling of the SUMO to the occupied $d_{\mathrm{z}^{2}}$ in the $\beta$ space $(\Delta \mathrm{E}=3.1 \mathrm{eV})$, dominates the contribution to the $y$ component $\left(\Delta \mathrm{g}_{\mathrm{y}}^{\mathrm{SO} / \mathrm{OZ}}\left(d_{\mathrm{z}^{2}} \leftrightarrow d_{\mathrm{xz}}^{*}\right)=+2086 \mathrm{ppt}\right)$, the total $y$ value being $\Delta \mathrm{g}_{\mathrm{y}}^{\mathrm{SO} / \mathrm{OZ}}=+2188 \mathrm{ppt}$ and the isotropic value $\Delta \mathrm{g}_{\text {iso }}^{\mathrm{SO} / \mathrm{OZ}}=+1021$ ppt for $\mathbf{1}$, see Figure 9 and Table $\mathrm{S} 7$, in accordance with our analysis in the section Theoretical Background.

The situation is completely different for compound $\mathbf{3}$. The formation of two strong $\pi$ bonds results in a notable destabilization of the corresponding $\pi^{*}\left(\operatorname{Ir}_{d_{\mathrm{xz}}}-\mathrm{N}_{p_{\mathrm{z}}}\right)$ and $\pi^{*}\left(\operatorname{Ir}_{d_{\mathrm{xy}}}-\mathrm{N}_{p_{\mathrm{y}}}\right)$ MOs (note in particular the latter in $\alpha$ space), so that the latter MO becomes vacant in compound $\mathbf{3}$ in contrast to that in $\mathbf{1}$, see Figure 5 . The SOMO can then couple very efficiently with the vacant $5 d_{\mathrm{xy}}$-based $\alpha$ spinor $(\Delta \mathrm{E}=2.8 \mathrm{eV})$ along the $x$ direction of the magnetic field $\left(\Delta \mathrm{g}_{\mathrm{x}}^{\mathrm{SO} / \mathrm{OZ}}\left(d_{\mathrm{xz}} \leftrightarrow d_{\mathrm{xy}}^{*}\right)=-597 \mathrm{ppt}\right)$, as shown in Figure 9, dominating the deshielding of the $\Delta \mathrm{g}_{\mathrm{x}}^{\mathrm{so} / \mathrm{Oz}}$ component (-532 ppt) and the corresponding isotropic $\Delta \mathrm{g}_{\text {iso }}^{\mathrm{SO} / \mathrm{OZ}}=-93 \mathrm{ppt}$ for $\mathbf{3}$, see Table $\mathrm{S} 7$.
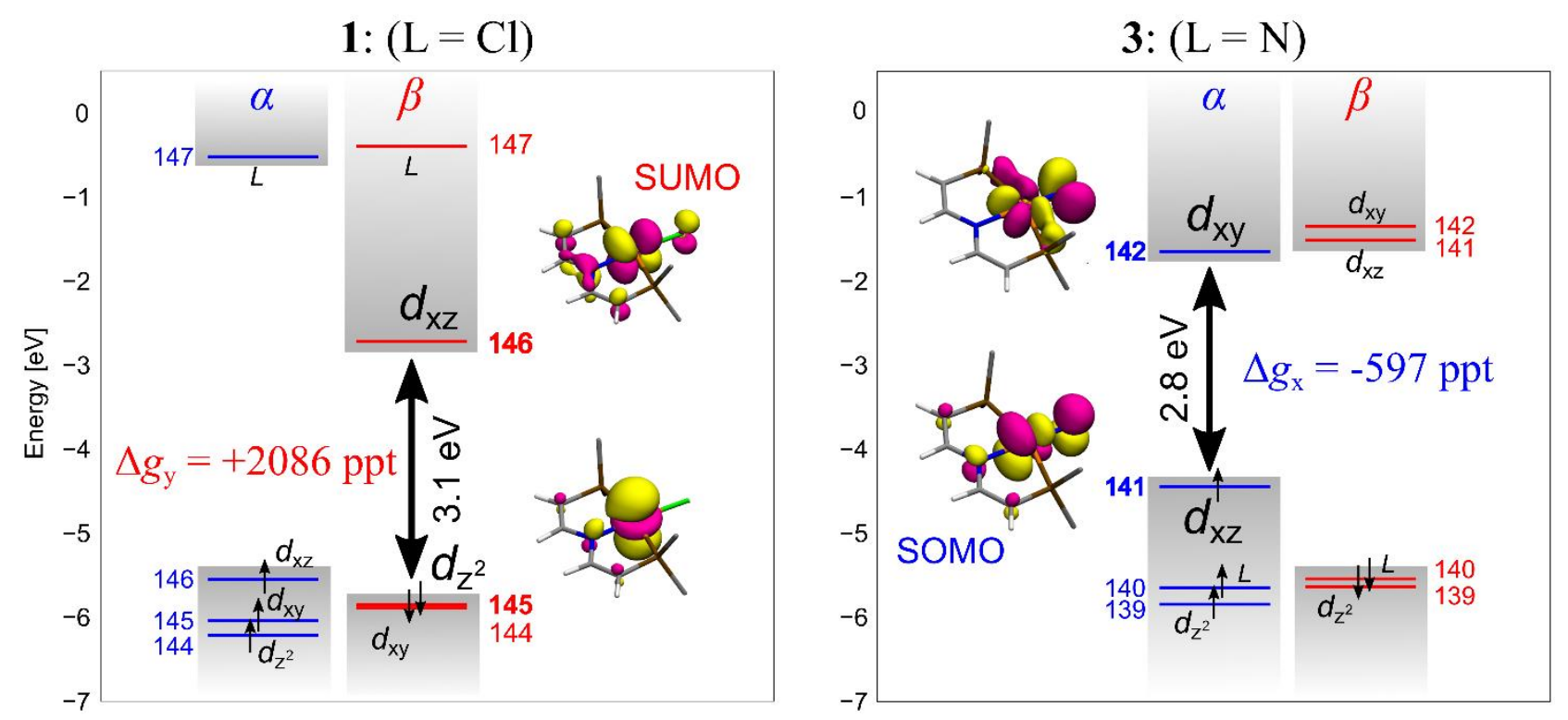

Figure 9. A portion of the molecular spin-orbital energy diagram and highlights from the PT2 excitation analysis of the contributions to the individual components of $\Delta \mathbf{g}^{\mathrm{SO} / \mathrm{OZ}}$ (PBE/vdz/upcJ1/vacuum) for compounds $\mathbf{1}$ (left) and $\mathbf{3}$ (right). 
As demonstrated in the section Theoretical Background, the $\mathbf{g}^{\mathrm{SO} / \mathrm{OZ}}$ and $\mathrm{A}^{\mathrm{SO} / \mathrm{PSO}}$ mechanisms have similarities in terms of the spin-orbit and magnetic-field operators, as summarized in Eqs. (2) and (5). We can therefore expect that the MSO couplings identified above for $\mathbf{g}$ also govern the trends in the PSO term of A.

\section{Ligand Hyperfine Coupling Tensor: Sign of A in Compounds 1 and 3.}

The total values of $A_{\text {iso }}$ for the atom $\mathrm{L}$ directly bonded to the metal $(\mathrm{Cl}$ in $1, \mathrm{~N}$ in 3$)$ are governed by the PSO terms, see Table 5. Similar observations have been reported previously for actinidecontaining systems with a large electronic orbital angular momentum. ${ }^{36,59,60}$ We highlight in particular the opposite signs of $A_{\text {iso }}^{\text {PSO }}(\mathrm{L})$ in compound $\mathbf{1}\left(+11.3 \mathrm{MHz}\right.$ for $\left.{ }^{35} \mathrm{Cl}\right)$ and $\mathbf{3}\left(-16.8 \mathrm{MHz}\right.$ for $\left.{ }^{14} \mathrm{~N}\right)$, despite both nuclei having positive gyromagnetic ratios. The opposite signs for $A$ thus parallel the $\Delta g$ values analyzed in the previous section. For the components of A, see Figure 10.

a)

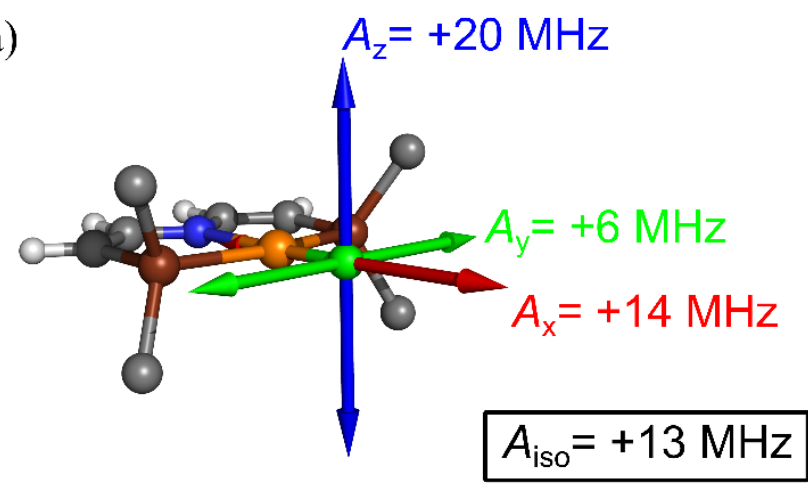

b)

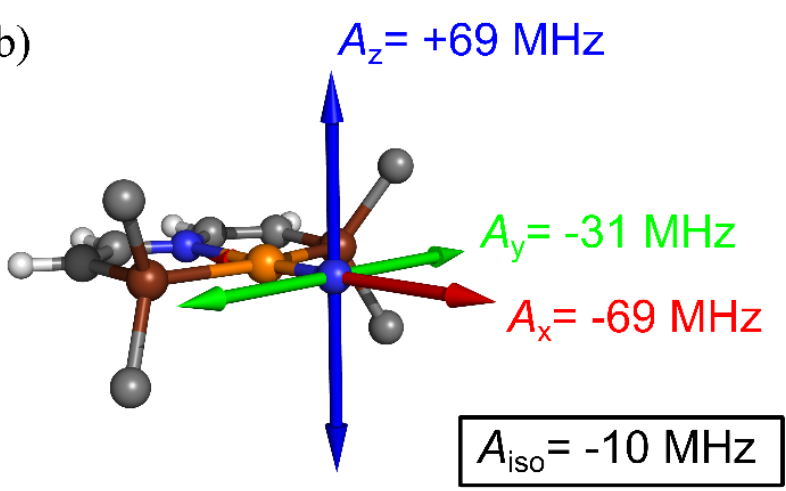

Figure 10. Visualization of components of the ligand L4 hyperfine A-tensor in molecular coordinate system for a) compound $\mathbf{1}$ and b) compound $\mathbf{3}$ calculated using the DKS approach (PBE0/vdz/upcJ$1 /$ vacuum). Note particularly the component $A_{\mathrm{x}}$, which shows the greatest changes (shown in red, +14 $\mathrm{MHz}$ for 1, -69 MHz for 3), oriented parallel to the Ir-L4 bond.

In compound 1 , the $A_{\mathrm{L}}^{\mathrm{PSO}}$-active coupling of the formal electron lone pair $\mathrm{n}\left(d_{z^{2}}\right)$ with the SUMO ( $\operatorname{Ir}_{d_{x z}}$-based) in $\beta$ space (see Figure 11) is clearly analogous to the mechanism of $\Delta \mathbf{g}$, vide supra. Note, however, that here it results in a negative contribution to $A_{\mathrm{y}}(-38 \mathrm{MHz})$, in contrast to that for $\Delta g_{\mathrm{y}}$. This is due to the different centers of the SO $\left(r_{M}^{-3} \hat{l}_{y}^{M}\right)$ and PSO $\left(r_{L}^{-3} \hat{l}_{y}^{L}\right)$ operators leading to the arbitrariness in the sign of the individual SO/PSO contributions. Also, the contribution of this $\mathrm{MO} \leftrightarrow \mathrm{MO}^{*}$ coupling to the overall $A_{\mathrm{L}}^{\mathrm{PSO}}$ is limited due to the minor admixture of atom $\mathrm{L}$ in the occupied $\beta \mathrm{MSO}$, as already noted. Instead, the coupling between the occupied $d_{\mathrm{xy}}$-based MSO and the SUMO in $\beta$ space dominates $A^{\mathrm{PSO}}$. This occupied-vacant $\pi^{*}\left(\operatorname{Ir}_{d_{x y}}-\mathrm{Cl}_{p_{y}}\right) \leftrightarrow \pi^{*}\left(\operatorname{Ir}_{d_{x z}}-\mathrm{Cl}_{p_{z}}\right)$ mixing due to the PSO operator is less important for $\Delta \mathbf{g}$, but is enhanced here due to the significant 
AO contributions of the chlorine atom (L4). This coupling, with a positive contribution to $A_{\mathrm{x}}(+66$ $\mathrm{MHz})$, is so efficient in the $A_{\mathrm{L}}^{\mathrm{PSO}}$ mechanism that is governs the sign not only of $A_{\mathrm{x}}(+59 \mathrm{MHz})$ but also of the total $A_{\text {iso }}(\mathrm{Cl} 4)$ value $(+15 \mathrm{MHz}$ PT2, $+14 \mathrm{MHz}$ DKS/PBE), see Table S7 in Supporting Information.

Energy destabilization of occupied $\alpha \mathrm{SOMO}, \pi^{*}\left(\operatorname{Ir}_{d_{x z}}-\mathrm{Cl}_{p_{z}}\right)$, and in particular the notable destabilization of the $d_{x y}$-based Ir-N4 antibonding MO with its shift to the vacant space, change completely the picture for compound $\mathbf{3}$, see Figure 11. In clear parallel to $\Delta \mathbf{g}$ for compound $\mathbf{3}$, $\pi^{*}\left(\operatorname{Ir}_{d_{\mathrm{xz}}}-\mathrm{Cl}_{p_{\mathrm{z}}}\right) \leftrightarrow \pi^{*}\left(\operatorname{Ir}_{d_{\mathrm{xy}}}-\mathrm{Cl}_{p_{\mathrm{y}}}\right)$ mixing upon the action of $\hat{l}_{x}^{L}(-52 \mathrm{MHz})$ dominates here the negative parallel component $A_{\mathrm{x}}(-54 \mathrm{MHz})$ as well as the total $A_{\text {iso }}(\mathrm{N} 4)$ value $(-17 \mathrm{MHz}$ PT2, $-10 \mathrm{MHz}$ DKS/PBE), see Table S7 in Supporting Information.
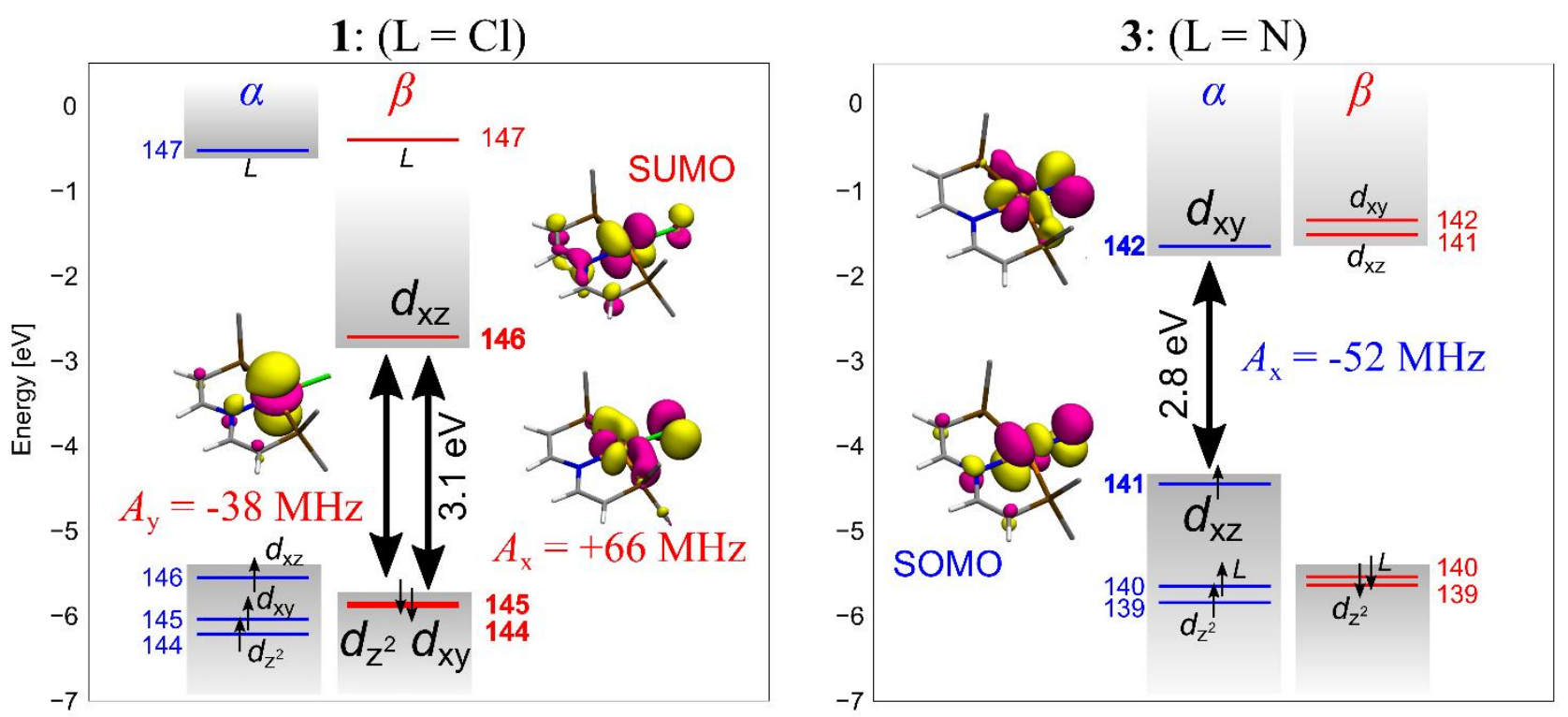

Figure 11. A portion of the molecular spin-orbital energy diagram and highlights from the PT2 excitation analysis of the contributions to the individual components of $\mathbf{A}^{\mathrm{SO} / \mathrm{PSO}}$ (PBE/vdz/upcJ1/vacuum) for compounds $\mathbf{1}$ (left) and $\mathbf{3}$ (right).

In summary, our analyses demonstrate that the different $\mathrm{M}-\mathrm{L}$ bonding situations in compound $\mathbf{1}$ vs $\mathbf{3}$ switches the energy of the $d_{\mathrm{xy}}-$ and $d_{\mathrm{xz}}$-based $\pi_{\mathrm{Ir}-\mathrm{N}}^{*}$ molecular spinors (MSOs) and thus also the dominant roles of the $\beta$ vs $\alpha$ MSOs in governing the electronic $\mathbf{g}$ and $\mathbf{A}_{\mathrm{L}}$ EPR parameters centered predominantly on the metal-ligand bond. The conclusions formulated about the relation between magnetic-resonance parameters and bonding characteristics are straightforwardly transferable to other systems, such as the very recently reported and structurally related compound $\operatorname{Ir}^{\mathrm{IV}}(\mathrm{PNP}) \mathrm{L} 4$ (terminal L4: $\left.=\mathrm{N}-{ }^{-} \mathrm{Bu}, g=1.555\right)^{61}$ or the analogous $\mathrm{Os}^{\mathrm{III}}(\mathrm{PNP}) \mathrm{Cl}_{2}$ complex $(g=1.812){ }^{62}$ This is easily further generalized for compounds of the d-block elements where early transition-metal 
complexes with low d-electron counts (e.g., $\left.\mathrm{d}^{1}\right)$ exhibit negative $\Delta g(\beta$ space), whereas very late transition-metal complexes (e.g., $\left.\mathrm{d}^{9}\right)$ typically have positive g-shifts ( $\alpha$ space). ${ }^{1}$ In the center of the $d$ block, one expects both spin spaces to contribute in the SO/OZ (SO/PSO) mechanisms $\left(\mathrm{SOMO}^{\alpha} \leftrightarrow \mathrm{MSO}^{\alpha *}, \mathrm{MSO}^{\beta} \leftrightarrow \mathrm{SUMO}^{\beta}\right)$ and the properties are fine-tuned by the ligands surrounding the central atoms. Because of the resemblance between the perturbation expressions for the $\Delta \mathrm{g}^{\mathrm{SO} / \mathrm{OZ}}$ and $\mathrm{A}^{\mathrm{SO} / \mathrm{PSO}}$ mechanisms, similar trends in future experimental determinations of the EPR ligand hyperfine couplings can be expected.

In a broad general-chemistry and reactivity context, our findings demonstrate that the EPR and NMR parameters reflect the positions of the SOMO and SUMO relative to the occupied and vacant metalbased MSOs, knowledge of which can be used to tune the electronic structure of a complex through chemical alterations, and in this way modify the reactivity of systems in catalytic reactions and the properties of molecular magnetic materials.

\section{CONCLUSIONS AND PROSPECTS}

We have performed a systematic study of the EPR parameters and paramagnetic NMR shifts for recently reported square-planar iridium complexes with the PNP pincer ligand. We have demonstrated how the opposite signs of the $g$-shift and ligand hyperfine $A$-coupling constant for the two complexes originate in the different electronic spin structure around the central iridium metal atom and differences in the metal-ligand bonding. This has significant consequences for the predictions of the paramagnetic NMR shifts which have no simple analogy in standard NMR spectroscopy of diamagnetic compounds. We have shown how the experimental NMR data can be assigned and interpreted using relativistic DFT calculations.

Based on second-order perturbation theory, we have provided a transparent interpretation of the shielding/deshielding contributions of $\alpha$ vs $\beta$ molecular spinors to the diagonal components of the $\mathrm{SO} / \mathrm{OZ}$ term of the electronic g-tensor. Furthermore, the close analogy between the metal-centered $\mathrm{SO} / \mathrm{OZ}$ mechanism of $\mathbf{g}$ and both the metal- and ligand- centered SO/PSO mechanism of $\mathbf{A}$ has been demonstrated.

The effects of solvent and relativity on magnetic resonance parameters were evaluated by using a fully relativistic DKS approach in combination with the PCM. We have shown how the electronic spin structure in terms of the metal oxidation state and the character of the metal-ligand bond can be mapped by relativistic EPR and NMR spectroscopy. The "through-bond" Fermi-contact contributions to the ligand hyperfine EPR coupling constants and ligand hyperfine NMR shifts are related to the calculated and visualized spin densities and spin populations. Whereas the Fermi-contact term is shown to dominate the hyperfine NMR shifts of most of the atoms in the systems investigated here, 
the hyperfine coupling of the ligand atom bonded directly to the iridium center (chlorine or nitrogen) is governed by the PSO term.

We have provided a clear and quantitative interpretation of all previously reported magnetic resonance observables using MO theory, in contrast to the statement in the original work regarding the challenges of performing a quantitative analysis of the EPR parameters in terms of the electronic structure when the system exhibits strong spin-orbit coupling. Our findings are straightforwardly transferable to other systems containing $d$-block elements and beyond. In a broader context, we have shown how EPR and NMR parameters can be rationalized using transparent chemical concepts that reflect the positions of the SOMO and SUMO energy levels (and symmetry) relative to the vacant and occupied metal-based MSOs. These orbitals can be efficiently tuned by coordinating ligands when developing catalytic and molecular magnetic materials.

The calculations and analysis of the hyperfine NMR shifts have enabled the assignment of previously reported NMR resonances in unusual shift ranges. As such, we have shown that relativistic DFT calculations can assist in the investigation and characterization of hitherto unknown exotic species. Further investigations of the relations between the electronic spin structure of open-shell systems and relativistic spin-orbit effects on the EPR and hyperfine NMR parameters are underway in our laboratories. 


\section{METHODS}

\section{Geometry Optimization.}

The starting geometry of compound $\mathbf{1}$ was taken from the X-ray crystal structure (CCDC number: Xray diffraction 822068, neutron diffraction 822069$)^{20}$ and re-optimized using Density-Functional Theory (DFT) with various unrestricted density functionals (UBP86, ${ }^{63-65} \mathrm{UBLYP}^{63}$ UB3LYP, ${ }^{66,67}$ CAM-UB3LYP, ${ }^{68} \mathrm{UPBE}^{69}{ }^{6} \mathrm{UPBE} 0,{ }^{41} \mathrm{UTPSSh}^{42,43}$ and UMN12SX ${ }^{44}$ with many of these additionally augmented by the D3 dispersion correction of Grimme ${ }^{70}$ with Becke-Johnson damping ${ }^{71}$, see Tables S2-S4 in Supporting Information) and the def2-TZVPP ${ }^{72}$ basis set for all atoms (if not stated otherwise), with corresponding relativistic effective core potentials (def2-ECPs) ${ }^{73}$ for the iridium atom (ECP substituting 60 core electrons), as implemented in the Turbomole 7.0 program. ${ }^{74}$ All calculations were performed using an $\mathrm{m} 5$ integration grid with the convergence criteria of $10^{-6}$ for the energy change and $10^{-3}$ for the geometry gradient. The structures were optimized either in vacuum or by using the COSMO (Conductor-like Screening Model) model of the solvent used in the EPR and NMR experiments (permittivity, $\varepsilon$, and probe radius, $\mathrm{r}_{\text {sol }}$, - toluene: $\varepsilon=2.38, \mathrm{r}_{\text {sol }}=3.48 \AA$; benzene: $\varepsilon$ $=2.30, \mathrm{r}_{\mathrm{sol}}=3.28 \AA$; tetrahydrofuran: $\varepsilon=7.58, \mathrm{r}_{\mathrm{sol}}=3.18 \AA$; diethyl ether: $\left.\varepsilon=4.34, \mathrm{r}_{\mathrm{sol}}=3.46 \AA\right){ }^{75}$

\section{Four-Component DFT Calculation of EPR and NMR Parameters.}

The magnetic resonance parameters (EPR and NMR) were calculated by using a developer version of the ReSpect program (version 4.0.0). ${ }^{76}$ The production calculations were performed at the fourcomponent Dirac-Kohn-Sham (DKS) level of theory. ${ }^{39,47,77-80}$ We tested the effects of solvent (vacuum vs PCM model), relativity (full DKS vs scalar-relativistic DKS approach employing downscaled $(\lambda=0)$ spin-orbit contribution, sc-DKS, - see below), and exact-exchange admixture in the hybrid PBE0 functional on the calculated EPR parameters. A detailed evaluation of the basis-set convergence and the effect of the density functional was performed in our recent work on paramagnetic $\mathrm{Ru}(\mathrm{III})$ compounds. ${ }^{30}$ We used basis sets of double- $\zeta$ quality: uncontracted Dyall's valence $\mathrm{v} d z$ for the metal ${ }^{81,82}$ and Jensen's uncontracted pcJ-1 (denoted as upcJ-1) for the light atoms. ${ }^{83,84}$ For the functional and basis-set dependencies tested in this work, see Figures S1-S2 in Supporting Information. The Mulliken spin populations and molecular spin-orbitals (MSOs) used in the figures were obtained by the scalar second-order Douglas-Kroll-Hess (sc-DKH2) calculations. ${ }^{85}$

To scale the SO contribution in the DKS framework, we multiply the 1-4 complex-quaternion constituents of the Fock matrix by a scaling factor $\lambda$. For the mapping of the Fock matrix to the complex quaternion algebra, see Eq. 23 in reference ${ }^{86} \cdot \lambda=0$ corresponds to the scalar fourcomponent picture and $\lambda=1$ represents the full four-component DKS calculation. 
Second-Order Perturbation Theory (PT2) - Analysis of the Spin-Orbit/Orbital-Zeeman Contribution to the $\mathbf{g}$-tensor, $\Delta \mathbf{g}^{\mathrm{SO} / \mathrm{OZ}}$, and the Spin-Orbit/Paramagnetic-Spin-Orbit (PSO) Contribution to the Atensor, $\boldsymbol{A}^{\mathrm{SO} / \mathrm{PSO}}$.

The calculation and analysis of the linear SO contributions to the g- and A-tensors were implemented into the ReSpect program package. For this purpose, the standard formulation of PT2 theory was used as described, for example, in references ${ }^{24,25,87}$. In this work, we made a few adjustments to the theory to render the expressions meaningful for an MO analysis. First, only the dominating one-electron SO operator is considered, neglecting the two-electron interactions. For systems containing one heavymetal center, the one-electron SO operator can be further restricted to $\sum_{N=1}^{\text {nuc }} Z^{N} r_{N}^{-3} \hat{\boldsymbol{l}}^{N} \approx Z^{M} r_{M}^{-3} \hat{\boldsymbol{l}}^{M}$, as the nuclear charge of the heavy atom $M$ is much larger than that of the light atoms. Second, we have performed the analysis using only pure DFT functionals, which allow us to avoid consideration of the DFT kernel otherwise present in calculations utilizing hybrid DFT functionals. Finally, the gauge of the angular momentum operator is placed on the atom $\mathrm{M}$ to decrease the basis set requirements, which results in fewer dominating MOs in Eq. (2).

The rationale behind Eq. (3) goes as follows: If we neglect the usually small interatomic contributions to the integrals in Eq. (2), the contribution from atomic orbitals (AOs) centered on the heavy atom M have the same sign for the SO, $\left\langle\varphi_{i}^{X}\left|r_{M}^{-3} \hat{\boldsymbol{l}}^{M}\right| \varphi_{a}^{X}\right\rangle$, and angular momentum, $\left\langle\varphi_{i}^{X}\left|\hat{\boldsymbol{l}}^{M}\right| \varphi_{a}^{X}\right\rangle$, integrals. Note that this holds only for the diagonal elements of the g-tensor, since the same component of the $\hat{\boldsymbol{l}}^{M}$ operator is then used in both integrals. Furthermore, the contributions of other AOs (e.g., the ligand atom in Figure 2) to the integral containing the angular momentum operator are not large enough to change the total sign. As a result, the sign in Eq. (2), the imaginary unit in the $\hat{\boldsymbol{l}}^{M}$ operator, the always negative denominator, and the antisymmetric relations of the integrals $\left(\left\langle\varphi_{i}^{X}\left|\hat{\boldsymbol{l}}^{M}\right| \varphi_{a}^{X}\right\rangle=-\left\langle\varphi_{a}^{X}\left|\hat{\boldsymbol{l}}^{M}\right| \varphi_{i}^{X}\right\rangle\right)$ determine the final sign of the $\alpha$ and $\beta$ electron contributions in Eq. (3).

\section{ACKNOWLEDGEMENTS}

This work has received support from the Czech Science Foundation (Grant No. 18-05421S), the Ministry of Education of the Czech Republic (Grant No. LQ1601), the SASPRO Program (Grant No. 1563/03/02), co-financed by the European Union and the Slovak Academy of Sciences, the Slovak Grant Agency APVV (Grant No. DS-2016-0009), and the Research Council of Norway through a Centre of Excellence Grant (Grant No. 262695). Computational resources were provided by the CESNET (Grant No. LM2015042), the CERIT Scientific Cloud (Grant No. LM2015085), and Norwegian supercomputing program NOTUR (Grant No. NN4654K). 


\section{ASSOCIATED CONTENT}

\section{Supporting Information}

The supporting information is available free of charge on the website at DOI: XXXXXX. Computational details, additional calculated EPR and NMR data and figures, and Cartesian coordinates (PDF).

\section{AUTHOR INFORMATION}

\section{Corresponding Author}

*E-mail: rmarek@chemi.muni.cz

\# P.L.B and J.N. contributed equally to this work.

\section{ORCID}

Jan Novotný: 0000-0002-1203-9549

Kenneth Ruud: 0000-0003-1006-8482

Stanislav Komorovsky: 0000-0002-5317-7200

Radek Marek: 0000-0002-3668-3523

\section{Notes}

The authors declare no competing financial interest. 


\section{REFERENCES}

(1) Mabbs, F. E.; Collison, D. In Electron Paramagnetic Resonance of d Transition Metal Compounds; Elsevier Science: Amsterdam, 1992; Vol. 16, pp 338-441.

(2) Symons, M. C. R. Chemical and Biochemical Aspects of Electron Spin Resonance Spectroscopy; Van Nostrand Reinhold Inc.,U.S.: New York, 1978.

(3) Mar, G. N. L.; Horrocks, W. D.; Holm, R. H. NMR of Paramagnetic Molecules: Principles and Applications; Elsevier: New York, 2013.

(4) Bertini, I.; Luchinat, C.; Parigi, G. Solution NMR of Paramagnetic Molecules: Applications to Metallobiomolecules and Models; Current Methods in Inorganic Chemistry; Elsevier Science: Amsterdam, 2016.

(5) Moon, S.; Patchkovskii, S. First-Principles Calculations of Paramagnetic NMR Shifts. In Calculation of NMR and EPR Parameters; Kaupp, M., Bühl, M., Malkin, V., Eds.; Wiley-VCH Verlag, 2004; pp 325-338.

(6) Autschbach, J. Chapter One - NMR Calculations for Paramagnetic Molecules and Metal Complexes. In Annual Reports in Computational Chemistry; Dixon, D. A., Ed.; Elsevier, 2015; Vol. 11, pp 3-36.

(7) Chirik, P. J.; Wieghardt, K. Radical Ligands Confer Nobility on Base-Metal Catalysts. Science 2010, 327, 794-795.

(8) Königsmann, M.; Donati, N.; Stein, D.; Schönberg, H.; Harmer, J.; Sreekanth, A.; Grützmacher, H. Metalloenzyme-Inspired Catalysis: Selective Oxidation of Primary Alcohols with an Iridium-Aminyl-Radical Complex. Angew. Chem. Int. Ed. 2007, 46, 3567-3570.

(9) Chan, K. S.; Li, X. Z.; Dzik, W. I.; de Bruin, B. Carbon-Carbon Bond Activation of 2,2,6,6Tetramethyl-Piperidine-1-Oxyl by a RhII Metalloradical: A Combined Experimental and Theoretical Study. J. Am. Chem. Soc. 2008, 130, 2051-2061.

(10) Puschmann, F. F.; Grützmacher, H.; Bruin, B. de. Rhodium(0) Metalloradicals in Binuclear C-H Activation. J. Am. Chem. Soc. 2010, 132, 73-75.

(11) Weststrate, C. J.; Bakker, J. W.; Gluhoi, A. C.; Ludwig, W.; Nieuwenhuys, B. E. Ammonia Oxidation on $\operatorname{Ir}(111)$ : Why Ir Is More Selective to N2 than Pt. Catal. Today 2010, 154, 46-52. (12) Grinberg Dana, A.; Elishav, O.; Bardow, A.; Shter, G. E.; Grader, G. S. Nitrogen-Based Fuels: A Power-to-Fuel-to-Power Analysis. Angew. Chem. Int. Ed Engl. 2016, 55, 8798-8805. (13) Buhr, J. D.; Taube, H. Oxidation of Pentaamminecarbonylosmium(2+) to $\mu$-DinitrogenBis(Cis-Tetraamminecarbonylosmium)(4+). Inorg. Chem. 1979, 18, 2208-2212.

(14) Ware, D. C.; Taube, H. Substitution-Induced Nitrogen-Nitrogen Coupling for Nitride Coordinated to Osmium(VI). Inorg. Chem. 1991, 30, 4605-4610.

(15) Lam, H.-W.; Che, C.-M.; Wong, K.-Y. Photoredox Properties of [OsN(NH3)4]3+ and Mechanism of Formation of $[\{\mathrm{Os}(\mathrm{NH} 3) 4(\mathrm{CH} 3 \mathrm{CN})\} 2 \mathrm{~N} 2] 5+$ through a Nitrido-Coupling Reaction. J. Chem. Soc. Dalton Trans. 1992, 0, 1411-1416.

(16) Demadis, K. D.; El-Samanody, E.-S.; Coia, G. M.; Meyer, T. J. OsIII(N2)OsII Complexes at the Localized-to-Delocalized, Mixed-Valence Transition. J. Am. Chem. Soc. 1999, 121, 535-544.

(17) Seymore, S. B.; Brown, S. N. Polar Effects in Nitride Coupling Reactions. Inorg. Chem. 2002, 41, 462-469.

(18) Man, W.-L.; Tang, T.-M.; Wong, T.-W.; Lau, T.-C.; Peng, S.-M.; Wong, W.-T. Highly Electrophilic (Salen)Ruthenium(VI) Nitrido Complexes. J. Am. Chem. Soc. 2004, 126, 478-479.

(19) Betley, T. A.; Peters, J. C. A Tetrahedrally Coordinated L3Fe-Nx Platform That Accommodates Terminal Nitride (FeIV:N) and Dinitrogen (FeI-N2-FeI) Ligands. J. Am. Chem. Soc. 2004, 126, 6252-6254.

(20) Meiners, J.; Scheibel, M. G.; Lemée-Cailleau, M.-H.; Mason, S. A.; Boeddinghaus, M. B.; Fässler, T. F.; Herdtweck, E.; Khusniyarov, M. M.; Schneider, S. Planar Iridium(II) and Iridium(III) Amido Complexes Stabilized by a PNP Pincer Ligand. Angew. Chem. Int. Ed. 2011, 50, 8184-8187. (21) Scheibel, M. G.; Askevold, B.; Heinemann, F. W.; Reijerse, E. J.; de Bruin, B.; Schneider, S. Closed-Shell and Open-Shell Square-Planar Iridium Nitrido Complexes. Nat. Chem. 2012, 4, 552- 
(22) Abragam, A.; Bleaney, B. Electron Paramagnetic Resonance of Transition Ions; Oxford Classic Texts in the Physical Sciences; Oxford University Press: Oxford, New York, 2012.

(23) Harriman, J. E. Theoretical Foundations of Electron Spin Resonance: Physical Chemistry: A Series of Monographs; Academic Press, 2013.

(24) Malkina, O. L.; Vaara, J.; Schimmelpfennig, B.; Munzarová, M.; Malkin, V. G.; Kaupp, M. Density Functional Calculations of Electronic G-Tensors Using Spin-Orbit Pseudopotentials and Mean-Field All-Electron Spin-Orbit Operators. J. Am. Chem. Soc. 2000, 122, 9206-9218.

(25) Rinkevicius, Z.; de Almeida, K. J.; Oprea, C. I.; Vahtras, O.; Ågren, H.; Ruud, K. Degenerate Perturbation Theory for Electronic g Tensors: Leading-Order Relativistic Effects. $J$. Chem. Theory Comput. 2008, 4, 1810-1828.

(26) Haase, P. A. B.; Repisky, M.; Komorovsky, S.; Bendix, J.; Sauer, S. P. A. Relativistic DFT Calculations of Hyperfine Coupling Constants in 5d Hexafluorido Complexes: [ReF6]2- and [IrF6]2-. Chem. - Eur. J. 2018, 24, 5124-5133.

(27) Novotný, J.; Přichystal, D.; Sojka, M.; Komorovsky, S.; Nečas, M.; Marek, R. Hyperfine Effects in Ligand NMR: Paramagnetic Ru(III) Complexes with 3-Substituted Pyridines. Inorg. Chem. 2018, 57, 641-652.

(28) Kaupp, M.; Köhler, F. H. Combining NMR Spectroscopy and Quantum Chemistry as Tools to Quantify Spin Density Distributions in Molecular Magnetic Compounds. Coord. Chem. Rev. 2009, 253, 2376-2386.

(29) Novotný, J.; Sojka, M.; Komorovsky, S.; Nečas, M.; Marek, R. Interpreting the Paramagnetic NMR Spectra of Potential Ru(III) Metallodrugs: Synergy between Experiment and Relativistic DFT Calculations. J. Am. Chem. Soc. 2016, 138, 8432-8445.

(30) Jeremias, L.; Novotný, J.; Repisky, M.; Komorovsky, S.; Marek, R. Interplay of ThroughBond Hyperfine and Substituent Effects on the NMR Chemical Shifts in Ru(III) Complexes. Inorg. Chem. 2018, 57, 8748-8759.

(31) Munzarová, M. L.; Kubáček, P.; Kaupp, M. Mechanisms of EPR Hyperfine Coupling in Transition Metal Complexes. J. Am. Chem. Soc. 2000, 122, 11900-11913.

(32) Cano, J.; Ruiz, E.; Alvarez, S.; Verdaguer, M. Spin Density Distribution in Transition Metal Complexes: Some Thoughts and Hints. Comments Inorg. Chem. 1998, 20, $27-56$.

(33) Chyba, J.; Novák, M.; Munzarová, P.; Novotný, J.; Marek, R. Through-Space Paramagnetic NMR Effects in Host-Guest Complexes: Potential Ruthenium(III) Metallodrugs with Macrocyclic Carriers. Inorg. Chem. 2018, 57, 8735-8747.

(34) Van den Heuvel, W.; Soncini, A. NMR Chemical Shift in an Electronic State with Arbitrary Degeneracy. Phys. Rev. Lett. 2012, 109, 073001.

(35) Heuvel, W. V. den; Soncini, A. NMR Chemical Shift as Analytical Derivative of the Helmholtz Free Energy. J. Chem. Phys. 2013, 138, 054113.

(36) Gendron, F.; Sharkas, K.; Autschbach, J. Calculating NMR Chemical Shifts for Paramagnetic Metal Complexes from First-Principles. J. Phys. Chem. Lett. 2015, 6, 2183-2188.

(37) Rouf, S. A.; Mareš, J.; Vaara, J. 1H Chemical Shifts in Paramagnetic Co(II) Pyrazolylborate Complexes: A First-Principles Study. J. Chem. Theory Comput. 2015, 11, 1683-1691.

(38) Vaara, J.; Rouf, S. A.; Mareš, J. Magnetic Couplings in the Chemical Shift of Paramagnetic NMR. J. Chem. Theory Comput. 2015, 11, 4840-4849.

(39) Komorovsky, S.; Repisky, M.; Ruud, K.; Malkina, O. L.; Malkin, V. G. Four-Component Relativistic Density Functional Theory Calculations of NMR Shielding Tensors for Paramagnetic Systems. J. Phys. Chem. A 2013, 117, 14209-14219.

(40) Bertini, I.; Luchinat, C.; Parigi, G. Magnetic Susceptibility in Paramagnetic NMR. Prog. Nucl. Magn. Reson. Spectrosc. 2002, 40, 249-273.

(41) Adamo, C.; Barone, V. Toward Reliable Density Functional Methods without Adjustable Parameters: The PBE0 Model. J. Chem. Phys. 1999, 110, 6158-6170.

(42) Perdew, J. P.; Tao, J.; Staroverov, V. N.; Scuseria, G. E. Meta-Generalized Gradient Approximation: Explanation of a Realistic Nonempirical Density Functional. J. Chem. Phys. 2004, 
$120,6898-6911$.

(43) Staroverov, V. N.; Scuseria, G. E.; Tao, J.; Perdew, J. P. Comparative Assessment of a New Nonempirical Density Functional: Molecules and Hydrogen-Bonded Complexes. J. Chem. Phys.

2003, 119, 12129-12137.

(44) Peverati, R.; Truhlar, D. G. Screened-Exchange Density Functionals with Broad Accuracy for Chemistry and Solid-State Physics. Phys. Chem. Chem. Phys. 2012, 14, 16187-16191.

(45) Vicha, J.; Novotny, J.; Straka, M.; Repisky, M.; Ruud, K.; Komorovsky, S.; Marek, R. Structure, Solvent, and Relativistic Effects on the NMR Chemical Shifts in Square-Planar Transition-Metal Complexes: Assessment of DFT Approaches. Phys. Chem. Chem. Phys. 2015, 17, 24944-24955.

(46) Gohr, S.; Hrobarik, P.; Repisky, M.; Komorovsky, S.; Ruud, K.; Kaupp, M. Four-Component Relativistic DFT Calculations of EPR g- and Hyperfine-Coupling Tensors Using Hybrid

Functionals: Validation on Transition-Metal Complexes with Large Tensor Anisotropies and HigherOrder Spin-Orbit Effects. J. Phys. Chem. A 2015, 119, 12892-12905.

(47) Remigio, R. D.; Repisky, M.; Komorovsky, S.; Hrobarik, P.; Frediani, L.; Ruud, K. FourComponent Relativistic Density Functional Theory with the Polarisable Continuum Model:

Application to EPR Parameters and Paramagnetic NMR Shifts. Mol. Phys. 2017, 115, 214-227.

(48) Burdett, J. K.; Albright, T. A. Trans Influence and Mutual Influence of Ligands Coordinated to a Central Atom. Inorg. Chem. 1979, 18, 2112-2120.

(49) Mitoraj, M. P.; Zhu, H.; Michalak, A.; Ziegler, T. On the Origin of the Trans-Influence in Square Planar D8-Complexes: A Theoretical Study. Int. J. Quantum Chem. 2009, 109, 3379-3386.

(50) Vícha, J.; Patzschke, M.; Marek, R. A Relativistic DFT Methodology for Calculating the Structures and NMR Chemical Shifts of Octahedral Platinum and Iridium Complexes. Phys. Chem. Chem. Phys. 2013, 15, 7740-7754.

(51) Novotný, J.; Vícha, J.; Bora, P. L.; Repisky, M.; Straka, M.; Komorovsky, S.; Marek, R. Linking the Character of the Metal-Ligand Bond to the Ligand NMR Shielding in Transition-Metal Complexes: NMR Contributions from Spin-Orbit Coupling. J. Chem. Theory Comput. 2017, 13, 3586-3601.

(52) Vícha, J.; Foroutan-Nejad, C.; Pawlak, T.; Munzarová, M. L.; Straka, M.; Marek, R. Understanding the Electronic Factors Responsible for Ligand Spin-Orbit NMR Shielding in Transition-Metal Complexes. J. Chem. Theory Comput. 2015, 11, 1509-1517.

(53) Matito, E.; Poater, J.; Solà, M.; Duran, M.; Salvador, P. Comparison of the AIM Delocalization Index and the Mayer and Fuzzy Atom Bond Orders. J. Phys. Chem. A 2005, 109, 9904-9910.

(54) Foroutan-Nejad, C.; Shahbazian, S.; Marek, R. Toward a Consistent Interpretation of the QTAIM: Tortuous Link between Chemical Bonds, Interactions, and Bond/Line Paths. Chem. - Eur. J. 2014, 20, 10140-10152.

(55) Mulliken, R. S. Electronic Population Analysis on LCAO-MO Molecular Wave Functions. I. J. Chem. Phys. 1955, 23, 1833-1840.

(56) Martin, B.; Autschbach, J. Kohn-Sham Calculations of NMR Shifts for Paramagnetic 3d Metal Complexes: Protocols, Delocalization Error, and the Curious Amide Proton Shifts of a HighSpin Iron(II) Macrocycle Complex. Phys. Chem. Chem. Phys. 2016, 18, 21051-21068.

(57) Pritchard, B.; Autschbach, J. Theoretical Investigation of Paramagnetic NMR Shifts in Transition Metal Acetylacetonato Complexes: Analysis of Signs, Magnitudes, and the Role of the Covalency of Ligand-Metal Bonding. Inorg. Chem. 2012, 51, 8340-8351.

(58) M. J. Frisch, G. W. Trucks, H. B. Schlegel, G. E. Scuseria, M. A. Robb, J. R. Cheeseman, G. Scalmani, V. Barone, G. A. Petersson, H. Nakatsuji, X. Li, M. Caricato, A. V. Marenich, J. Bloino, B. G. Janesko, R. Gomperts, B. Mennucci, H. P. Hratchian, J. V. Ortiz, A. F. Izmaylov, J. L. Sonnenberg, D. Williams-Young, F. Ding, F. Lipparini, F. Egidi, J. Goings, B. Peng, A. Petrone, T. Henderson, D. Ranasinghe, V. G. Zakrzewski, J. Gao, N. Rega, G. Zheng, W. Liang, M. Hada, M. Ehara, K. Toyota, R. Fukuda, J. Hasegawa, M. Ishida, T. Nakajima, Y. Honda, O. Kitao, H. Nakai, T. Vreven, K. Throssell, J. A. Montgomery, Jr., J. E. Peralta, F. Ogliaro, M. J. Bearpark, J. J. Heyd, 
E. N. Brothers, K. N. Kudin, V. N. Staroverov, T. A. Keith, R. Kobayashi, J. Normand, K. Raghavachari, A. P. Rendell, J. C. Burant, S. S. Iyengar, J. Tomasi, M. Cossi, J. M. Millam, M. Klene, C. Adamo, R. Cammi, J. W. Ochterski, R. L. Martin, K. Morokuma, O. Farkas, J. B. Foresman, and D. J. Fox. Gaussian 16; Gaussian, Inc., Wallingford CT.

(59) Gendron, F.; Autschbach, J. Ligand NMR Chemical Shift Calculations for Paramagnetic Metal Complexes: 5f1 vs 5f2 Actinides. J. Chem. Theory Comput. 2016, 12, 5309-5321.

(60) Sergentu, D.-C.; Gendron, F.; Autschbach, J. Similar Ligand-Metal Bonding for Transition Metals and Actinides? 5f1 U(C7H7)2-versus 3dn Metallocenes. Chem. Sci. 2018, 9, 6292-6306.

(61) Kinauer, M.; Diefenbach, M.; Bamberger, H.; Demeshko, S.; Reijerse, E. J.; Volkmann, C.; Würtele, C.; Slageren, J. van; Bruin, B. de; Holthausen, M. C.; et al. An Iridium(III/IV/V) Redox Series Featuring a Terminal Imido Complex with Triplet Ground State. Chem. Sci. 2018, 9, 43254332.

(62) Abbenseth, J.; Diefenbach, M.; Bete, S. C.; Würtele, C.; Volkmann, C.; Demeshko, S.; Holthausen, M. C.; Schneider, S. A Square-Planar Osmium(II) Complex. Chem. Commun. 2017, 53, 5511-5514.

(63) Becke, A. D. Density-Functional Exchange-Energy Approximation with Correct Asymptotic Behavior. Phys. Rev. A 1988, 38, 3098-3100.

(64) Perdew, J. P. Density-Functional Approximation for the Correlation Energy of the Inhomogeneous Electron Gas. Phys. Rev. B 1986, 33, 8822-8824.

(65) Perdew, J. P. Erratum: Density-Functional Approximation for the Correlation Energy of the Inhomogeneous Electron Gas. Phys. Rev. B 1986, 34, 7406-7406.

(66) Becke, A. D. Density-functional Thermochemistry. III. The Role of Exact Exchange. $J$. Chem. Phys. 1993, 98, 5648-5652.

(67) Stephens, P. J.; Devlin, F. J.; Chabalowski, C. F.; Frisch, M. J. Ab Initio Calculation of Vibrational Absorption and Circular Dichroism Spectra Using Density Functional Force Fields. $J$. Phys. Chem. 1994, 98, 11623-11627.

(68) Yanai, T.; Tew, D. P.; Handy, N. C. A New Hybrid Exchange-Correlation Functional Using the Coulomb-Attenuating Method (CAM-B3LYP). Chem. Phys. Lett. 2004, 393, 51-57.

(69) Perdew, J. P.; Burke, K.; Ernzerhof, M. Generalized Gradient Approximation Made Simple. Phys. Rev. Lett. 1996, 77, 3865-3868.

(70) Grimme, S.; Antony, J.; Ehrlich, S.; Krieg, H. A Consistent and Accurate Ab Initio Parametrization of Density Functional Dispersion Correction (DFT-D) for the 94 Elements H-Pu. $J$. Chem. Phys. 2010, 132, 154104.

(71) Grimme, S.; Ehrlich, S.; Goerigk, L. Effect of the Damping Function in Dispersion Corrected Density Functional Theory. J. Comput. Chem. 2011, 32, 1456-1465.

(72) Rappoport, D.; Furche, F. Property-Optimized Gaussian Basis Sets for Molecular Response Calculations. J. Chem. Phys. 2010, 133, 134105.

(73) Andrae, D.; Häußermann, U.; Dolg, M.; Stoll, H.; Preuß, H. Energy-Adjusted Ab Initio Pseudopotentials for the Second and Third Row Transition Elements. Theor. Chim. Acta 1990, 77, 123-141.

(74) TURBOMOLE V7.0 2015, a Development of University of Karlsruhe and Forschungszentrum Karlsruhe GmbH, 1989-2007, TURBOMOLE GmbH, since 2007; Available from Http://Www.Turbomole.Com.

(75) Klamt, A.; Schüürmann, G. A New Approach to Dielectric Screening in Solvents with Explicit Expressions for the Screening Energy and Its Gradient. J. Chem. Soc. Perkin Trans. 2 1993, 0, 799-805.

(76) Repisky, M.; Komorovsky, S.; Malkin, V. G.; Malkina, O. L.; Kaupp, M.; Ruud, K.; Bast, R.; Ekstrom, U.; Kadek, M.; Knecht, S.; Konečn y, L.; Malkin, E.; Malkin Ondík, I. Relativistic Spectroscopy DFT Program ReSpect, Developer Version 4.0.0; 2017. Www.Respectprogram.Org. (77) Repisky, M.; Komorovsky, S.; Malkin, E.; Malkina, O. L.; Malkin, V. G. Relativistic FourComponent Calculations of Electronic g-Tensors in the Matrix Dirac-Kohn-Sham Framework. Chem. Phys. Lett. 2010, 488, 94-97. 
(78) Malkin, E.; Repisky, M.; Komorovsky, S.; Mach, P.; Malkina, O. L.; Malkin, V. G. Effects of Finite Size Nuclei in Relativistic Four-Component Calculations of Hyperfine Structure. J. Chem. Phys. 2011, 134, 044111.

(79) Komorovský, S.; Repiský, M.; Malkina, O. L.; Malkin, V. G.; Malkin Ondík, I.; Kaupp, M. A Fully Relativistic Method for Calculation of Nuclear Magnetic Shielding Tensors with a Restricted Magnetically Balanced Basis in the Framework of the Matrix Dirac-Kohn-Sham Equation. J. Chem. Phys. 2008, 128, 104101.

(80) Komorovsky, S.; Repisky, M.; Malkina, O. L.; Malkin, V. G. Fully Relativistic Calculations of NMR Shielding Tensors Using Restricted Magnetically Balanced Basis and Gauge Including Atomic Orbitals. J. Chem. Phys. 2010, 132, 154101.

(81) Dyall, K. G. Relativistic Double-Zeta, Triple-Zeta, and Quadruple-Zeta Basis Sets for the 5d Elements Hf-Hg. Theor. Chem. Acc. 2004, 112, 403-409.

(82) Dyall, K. G.; Gomes, A. S. P. Revised Relativistic Basis Sets for the 5d Elements Hf-Hg. Theor. Chem. Acc. 2010, 125, 97.

(83) Jensen, F. Polarization Consistent Basis Sets: Principles. J. Chem. Phys. 2001, 115, 91139125.

(84) Jensen, F.; Helgaker, T. Polarization Consistent Basis Sets. V. The Elements Si-Cl. J. Chem. Phys. 2004, 121, 3463-3470.

(85) Reiher, M. Relativistic Douglas-Kroll-Hess Theory. Wiley Interdiscip. Rev. Comput. Mol. Sci. 2012, 2, 139-149.

(86) Konecny, L.; Kadek, M.; Komorovsky, S.; Ruud, K.; Repisky, M. Resolution-of-Identity Accelerated Relativistic Two- and Four-Component Electron Dynamics Approach to Chiroptical Spectroscopies. J. Chem. Phys. 2018, accepted.

(87) Arbuznikov, A. V.; Vaara, J.; Kaupp, M. Relativistic Spin-Orbit Effects on Hyperfine Coupling Tensors by Density-Functional Theory. J. Chem. Phys. 2004, 120, 2127-2139. 